This item was submitted to Loughborough's Research Repository by the author.

Items in Figshare are protected by copyright, with all rights reserved, unless otherwise indicated.

\title{
Damping of flexural vibrations in circular plates with tapered central holes
}

PLEASE CITE THE PUBLISHED VERSION

http://dx.doi.org/10.1016/j.jsv.2010.11.017

\section{PUBLISHER}

(c) Elsevier Ltd.

\section{VERSION}

SMUR (Submitted Manuscript Under Review)

LICENCE

CC BY-NC-ND 4.0

\section{REPOSITORY RECORD}

O'Boy, D.J., and Victor V. Krylov. 2012. "Damping of Flexural Vibrations in Circular Plates with Tapered Central Holes". figshare. https://hdl.handle.net/2134/9957. 
This item was submitted to Loughborough's Institutional Repository (https://dspace.lboro.ac.uk/) by the author and is made available under the following Creative Commons Licence conditions.

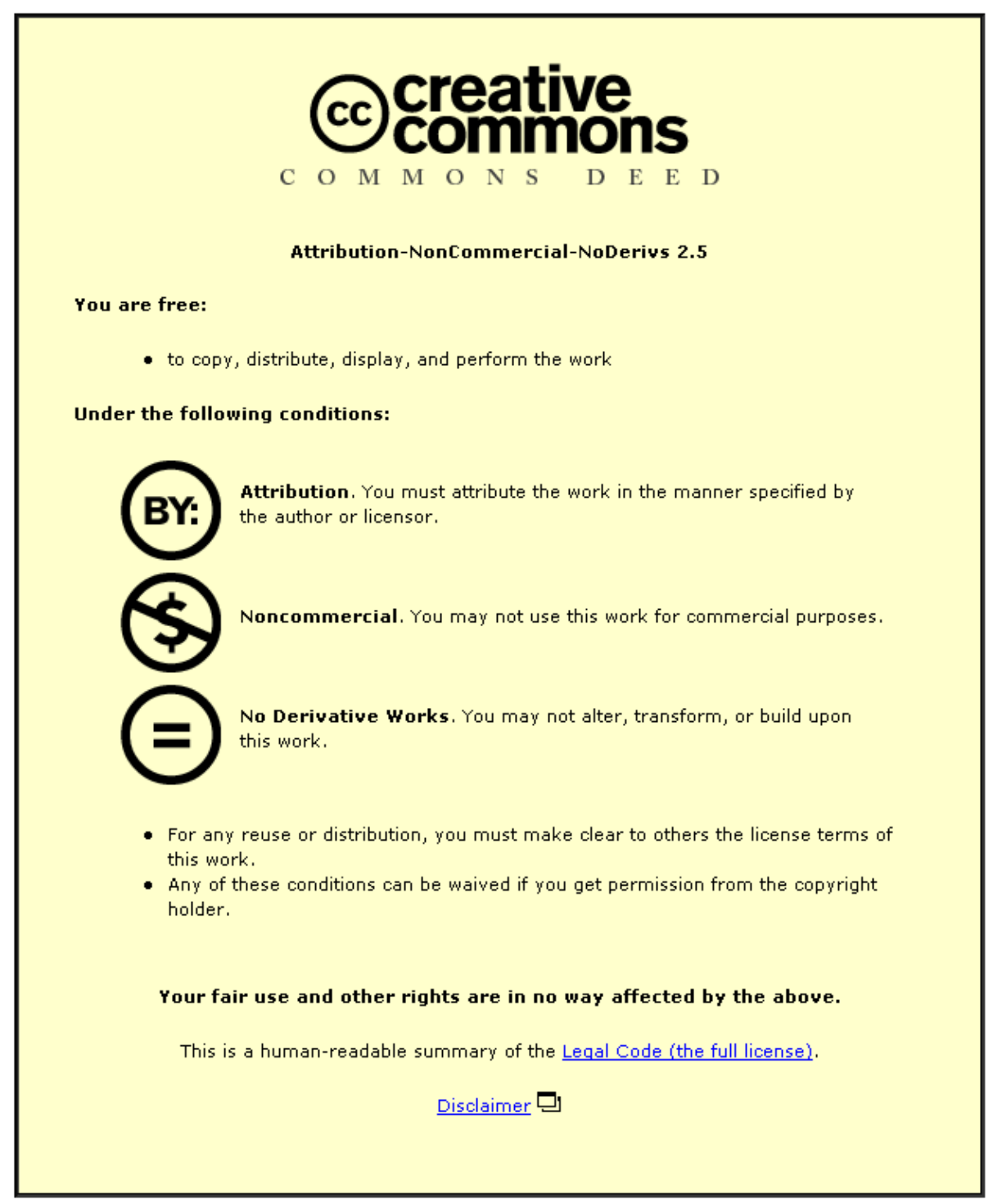

For the full text of this licence, please go to: http://creativecommons.org/licenses/by-nc-nd/2.5/ 


\title{
Damping of flexural vibrations in circular plates with tapered central holes
}

\author{
D. J. O’Boy* V. V.Krylov \\ Department of Aeronautical and Automotive Engineering, \\ Loughborough University, \\ Loughborough, Leicestershire, LE11 3TU, UK
}

\begin{abstract}
The paper develops a numerical approach to the calculation of mobilities for a circular plate with a tapered central hole of power-law profile. The exact solution of the corresponding flexural wave equation that exists for $m=2$ has been used in the process of the numerical solution of the corresponding boundary problem. Note that this value of $m$ belongs to the power-law range $m \geq 2$ associated with zero reflection of quasi-plane waves from a tapered hole in geometrical acoustics approximation. Two cases of added damping in the central hole area have been considered: a thin absorbing layer and a constrained layer. Cross and point mobilities have been calculated for both these cases. The obtained results for point and cross mobilities show a substantial suppression of resonant peaks (up to $17 \mathrm{~dB}$ ), in comparison with the cases of a plate with an uncovered hole of the same power-law profile and of a reference circular plate of constant thickness covered or uncovered by a thin absorbing layer. Further theoretical and experimental research is needed to examine applications of the obtained numerical results to more practical situations, e.g. to rectangular plates or other structures with arbitrary locations of tapered holes.

Key words: Vibration damping, Circular plates, Tapered holes
\end{abstract}




\section{Introduction}

The knowledge of plate vibrations is of key importance in the design of structures. The reduction of unwanted vibrations is important for structural security, stability and comfort (both tactile and acoustic). This reduction can be accomplished through both manipulation of the structure to move natural frequencies of vibration out of the range of operating frequencies or the damping of existing plate vibrations. There are numerous examples of practical methods and commercial products used to alter the natural frequencies of plates, reduce the vibration at key frequencies or attenuate vibrations at certain frequency ranges.

There has been significant research into the natural frequencies of vibration of constant thickness plates, of different shapes and materials, of which the development of simple bending plate models has been critical. Various common boundary conditions have been represented to determine mode shapes and resonances of simple shapes [1][2]. Many of these are based on analytical methods, as the equations of motion are relatively simple to solve, some are based on numerical methods for complex or mixed boundary conditions and experimental measurements where methods such as finite elements are not practical.

\footnotetext{
* Corresponding author.

Email address: d.j.oboy@lboro.ac.uk (D. J. O'Boy).

URL: http://www.lboro.ac.uk/departments/tt/staff/oboy.html (D. J. O'Boy).
} 
There are fewer examples of work conducted into the vibrations of cylindrical plates where the thickness varies according to the radius, primarily due to the increased complexity of the equations of motion restricting the availability of analytical solutions.

The thickness, $h(r)$, of the plates examined in the literature vary with the plate radius according to a linear, polynomial or exponential variation. All tend to introduce a taper coefficient $\epsilon$ which determines the thickness variation with $r$ such that $h(r)=\epsilon r^{m}$, where $m$ can be a positive or negative real number (from an initial thickness $h_{0}$ ). For the former, the plate is thinner at the centre of the plate. The linear variation occurs when $m= \pm 1$.

There are even fewer examples of work conducted into the advantages of damping variable thickness plates. This is despite authors specialising in damping vibrations recognising at an early stage that the thickness and properties of the damping layer relative to the base plate are of key importance [3]. However, recent investigations have taken place which have shown the potential advantages (for vibration reductions) of tapered plates and beams of specified profile [4][5]. These investigations have shown the potential to reduce the amplitude of vibration of beams and rectangular plates by including a wedge where the thickness of the plate reduces from $h_{0}$ to zero in a short distance, $x$, from one edge such that $h(x)=\epsilon x^{m}$.

It can be shown that a flexural wave of single frequency moving through a wedge which has a reduction in thickness slows down and grows in amplitude, an exchange which is governed by the conservation of energy in a small control volume. According to the geometrical-acoustics theory developed in [4][5], a wave travelling towards a wedge of profile $m \geq 2$ would asymptotically slow 
down, never reach the end of the wedge and therefore could never reflect back. The reflection coefficient from the free edge then becomes zero, when $m \geq 2$. In practical terms, the main problem in utilising this effect is that a truncation must be included to the wedge, as the material thickness is eventually stopped by the limitations of the machining process. Then reflection occurs from the free edge and the wave moves back towards the plate. Krylov has suggested using small amounts of damping material at the leading edge to attenuate the waves as much as possible (see [4][5]), with success as demonstrated in Krylov et al. for elastic plates of power-law profile [6] and in O'Boy et al. [7] for rectangular plates with attached wedges.

In this paper, we consider a numerical model of profiled cylindrical holes inside cylindrical plates of constant thickness, to quantify the potential reduction in resonant vibration amplitudes which are possible to obtain in this twodimensional geometry. Note that two-dimensional power-law holes for plate bending waves have been recently considered by Krylov [8] using geometrical acoustics theory. Their successful application for damping resonant vibrations in elliptical plates has been demonstrated experimentally by Gautier et al. [9]. In the present paper we develop a wave model of a cylindrical plate with a small wedge-like profiled hole located in the centre that follows a quadratic power-law design.

This paper contains a description of a numerical model which can predict the natural frequencies and mode shapes of vibration of a cylindrical plate with and without a wedge-like hole, as well as the associated frequency response function. Section 3 contains a description of the model, with details of the implementation given in section 4 . This is then used in section 5 to provide 
a comparison between three different plates; i) plain circular plate of outer radius $r=R_{o}$, ii) circular annulus plate with outer radius $r=R_{o}$ and inner radius $R_{i}$, iii) a circular plate of outer radius $r=R_{o}$ with a tapered hole at the centre.

The wedge-like hole which is incorporated into the plate has a local thickness $h(r)$ which is given by $h(r)=\epsilon r^{2}$, as the previous work [8] shows that the near zero reflection coefficient from the free edge starts when the power-law is $m=2$. This quadratic power-law also allows the use of an analytical solution to the bending plate equation of motion. An illustration of the composite plate is provided in Fig. 1 where a small cross section has been removed to show the change in thickness with a variation in radial position.

Three different damping configurations are demonstrated; i) Material damping inherent in all elastic materials, ii) a thin constant layer thickness damping applied over the wedge, iii) A thick damping layer which fills the wedge hole and is constrained by another thin layer on the top surface such that the exterior of the plate is smooth.

Conclusions are presented in section 6 . The overall aim of the paper is to demonstrate the viable potential for damping a circular plate with a circular wedge-like hole of profile $m=2$. The paper uses a single excitation and response method (an arbitrary position) to examine the plate response for different damping configurations. For a single frequency this point may line up with vibration nodes and anti-nodes, which are especially problematic at lower frequencies. Therefore the analysis is carried out over a broad frequency range and the results analysed over many resonant peaks to mitigate against this effect, however with a single arbitrary point measurement, it is not pos- 
sible to determine the overall loss factor in the whole plate, therefore this is not carried out.

\section{Existing solutions for cylindrical plates}

This section contains a brief literature review for vibrations of cylindrical plates, detailing the numerous approximations which have been used to solve some of the equations of motion.

A number of specific instances where analytical solutions for circular plate vibration are available have been provided by Conway [10], who finds a solution which varies according to the profiles $m=2 / 3$ and $m=18 / 21$, but only when the plate material has a Poisson's ratio $\nu=1 / 9$ and $\nu=5 / 21$ respectively, limiting its applicability. A linear variation is also presented with $m=1$ and $\nu=0.3$, close to the properties of steel. A more arbitrary solution is used in this paper, that of the profile $m=2$ with arbitrary Poisson's ratio.

A numerical solution is provided by Harris [11] for a plate where the thickness tapers parabolically $(m=2)$ to zero at the outer radius. Harris utilises a series expansion for the displacement term and an arrangement of a series of infinite matrices to determine an infinite number of natural frequencies and corresponding mode shapes across the radius of the plate. As this is a purely numerical solution, it does not depend on restrictions to the value of Poisson's ratio, however Harris states that the solution becomes less dependent on this ratio with increasing frequency.

The use of series expansions in the displacement function is more common. For example, Jain [12] has also studied the same plate form as Harris, how- 
ever he provides a greater detail on the convergence of the infinite series and factors which influence the lowest mode shapes. He has included an in-plane force in the plate and studied the natural frequencies for clamped and simply supported boundary conditions.

The inclusion of in-plane forces and also elastic restraints was studied by both Laura [13] and Gupta [14][15]. The latter used a series solution of the displacement function when the taper coefficient is altered. The inclusion of the elastic restraints is important in the formulation as it more accurately reflects practical applications of plate structures, rather than the more idealised simply supported or clamped boundary conditions more usually assumed. The solutions are provided for the case where the thickness varies linearly and also parabolically. The method by the group led by Laura is nominally for a linear variation in thickness, however, they use a polynomial approach to approximate the displacement function and then implement Galerkin's method to minimise the error between this approximation and the differential equation. Since this is a general numerical method, it can be used for any $m$ with any value of the taper coefficient (subject to computational limits).

Another numerical approach to determining the natural frequencies of vibration is to use the Rayleigh-Ritz method, for example see Singh [16] who finds the first four frequencies and nodal radii for a plate with an exponential thickness variation. No discussion on convergence is given or limits on applicability other than to say that the integrals involved must be completed accurately. Comparisons are made with a constant thickness plate and a linear variation. Other authors using the Rayleigh-Ritz method include Singh [17], Gupta [18] and Taher [19], where in the latter case the accuracy was determined through comparisons with finite element calculations. 
A less common approach has been given by Elishakoff [20], who rather than specifying the form of the numerical displacement on the plate, instead specifies a plate vibration mode and then determines a stiffness function which leads to the natural frequency (named the inverse problem). This has been used with success on several profiles of cylindrical plates including a parabolic thickness variation where the outer plate radius is clamped as a boundary condition.

The complexity of the equation of motion for variable thickness plates can be simplified by assuming that the thickness varies slowly with a change in radius. Then, perturbation theory allows the displacement function to be separated into a mean and small perturbation component. Yang proposes this method and uses it to solve the case of a linear wedge shape, with comparisons to finite element results [21]. Yang finds that although the method is available for arbitrary $m$, the use at higher taper coefficients leads up to a 6.5 percent variation in natural frequencies.

A final example of a numerical method is by Wang [22], who uses a differential quadrature (DQ) method to evaluate the natural frequencies of vibration of a plate whose thickness varies from $m= \pm 1$. This method allows highly accurate solutions to differential equations to be found using only a small number of grid points. Wang uses prescribed rotational stiffnesses on the plate edges to realise boundary conditions from free edges to completely clamped.

Most of the authors represented above do not discuss limitations of their solutions other than stating that at times, the solutions are singular at $r=0$ (and are therefore not considered), nor do they consider applications of tapering in circular plates to damping structural vibrations (through an analysis of the 
amplitude of modal displacement). All of these aspects will be considered in the following sections of the present paper.

\section{Numerical model}

In order to be able to predict the vibration of a circular plate with and without a profiled wedge-like hole, two separate sections are required. With reference to Fig. 2, two constant thickness discs are created, joined at a specified radius. At this join a boundary condition force is applied to excite the structure. At the centre of the disc, a profiled section can be included, which terminates at a truncation radius.

For notation, the outer radius of the first constant thickness plate is $R_{o}$, the inner radius, $R_{f}$ (this is where the force will be applied) and thickness $h$, density $\rho$, Young's modulus $E$ and Poisson's ratio $\nu$. The second constant thickness plate has outer radius $R_{f}$ and inner radius $R_{i}$, the vibration is measured at a radius $R_{m}$ with the same material properties as previously described.

At the centre of the composite plate is a power-law profiled hole, hereby referred to as the wedge-like hole with subscript $w$. The outer radius is $R_{i}$ and inner radius $R_{t}$ (the truncation position) with material properties $E_{w}, \rho_{w}$ and $\nu_{w}$.

The initial solution is determined assuming a vertical plate displacement $w(r, \theta, t)=w(r) e^{\mathrm{i} n \theta} e^{-\mathrm{i} \omega t}$. The variables $\theta$ and $t$ are the circumferential angle and time respectively. Fourier transforms can be taken to express the displacement as functions of the angular order $n$ and frequency $\omega$. The displacement of the wedge-like section is denoted $w_{w}(r, \theta, \omega)$. The forward Fourier transform 
and subsequent inversion are defined as,

$$
\begin{aligned}
\tilde{w}(r, n, \omega) & =\frac{1}{(2 \pi)^{2}} \int_{-\infty}^{\infty} \int_{-\pi}^{\pi} w(r, \theta, t) e^{-\mathrm{i} n \theta} e^{\mathrm{i} \omega t} \mathrm{~d} \theta \mathrm{d} t \\
w(r, \theta, t) & =\int_{-\infty}^{\infty} \sum_{n=-\infty}^{\infty} \tilde{w}(r, n, \omega) e^{\mathrm{i} n \theta} e^{-\mathrm{i} \omega t} \mathrm{~d} \omega
\end{aligned}
$$

The damping is applied as a complex Young's modulus, so that $E=E(1+\mathrm{i} \eta)$, where $\eta$ is the loss factor as has been used in previous studies [7]. The loss factor associated with the wedge-like section material is $\eta_{w}$, where it is assumed that the mass of the damping layer is not significant in comparison to the wedge material. In addition, the loss factor applied to the wedge is assumed not to vary with frequency, although the implementation of the numerical method means that any frequency dependent damping could be applied.

\subsection{Circular plate of constant thickness}

The vibration of an isotropic, constant thickness radial plate is well documented, see for example [1][2]. A short description is provided here for completeness.

The equation of motion of a constant thickness annulus is given by [12],

$$
-D \nabla^{4} w(r, \theta, t)=\rho h \frac{\partial^{2} w(r, \theta, t)}{\partial t^{2}}
$$

where $D=E h^{3} / 12\left(1-\nu^{2}\right)$ is the bending stiffness and $\nabla^{4}$ is the differential operator in polar coordinates,

$$
\nabla^{2} \nabla^{2}=\left(\frac{\partial^{2}}{\partial r^{2}}+\frac{1}{r} \frac{\partial}{\partial r}+\frac{1}{r^{2}} \frac{\partial^{2}}{\partial \theta^{2}}\right)\left(\frac{\partial^{2}}{\partial r^{2}}+\frac{1}{r} \frac{\partial}{\partial r}+\frac{1}{r^{2}} \frac{\partial^{2}}{\partial \theta^{2}}\right)
$$


We apply the form of the solution for displacement to the equation of motion,

$$
D \nabla^{4} w(r, \theta)-\rho h \omega^{2} w(r, \theta)=0 .
$$

Now defining $\beta^{4}=\rho h \omega^{2} / D$, so that the equation becomes $D \nabla^{4} w(r, \theta)-$ $\beta^{4} w(r, \theta)=0$. We then write the total displacement as the sum of two distinct solutions, $w(r, \theta)=w_{1}+w_{2}$ where,

$$
\nabla^{2} w_{1}+\beta^{2} w_{1}=0, \quad \nabla^{2} w_{2}-\beta^{2} w_{2}=0 .
$$

If $w_{1}=f(r) e^{\mathrm{i} n \theta}$ then after substitution into the above it can be shown that a solution exists in terms of Bessel's functions with constants $c_{1}$ and $c_{2}$.

$$
w_{1}=\left(c_{1} J_{n}(\beta r)+c_{2} Y_{n}(\beta r)\right) e^{\mathrm{i} n \theta} e^{-\mathrm{i} \omega t} .
$$

Similarly, the alternative solution includes modified Bessel's functions, $I_{n}(\beta r)=$ $\left.J_{n}(\mathrm{i} \beta r)\right)$,

$$
w_{2}=\left(c_{3} I_{n}(\beta r)+c_{4} K_{n}(\beta r)\right) e^{\mathrm{i} n \theta} e^{-\mathrm{i} \omega t} .
$$

For a solid cylindrical disc of constant cross section, the constants $c_{2}$ and $c_{4}$ are zero, as the corresponding Bessel's functions are singular at $r=0$.

This standard solution to the vibration displacement of a circular plate contains four constants. The model presented in this paper utilises two such constant thickness plates, therefore we have eight constants to determine. Four of these will immediately be satisfied as the two circular surfaces are common, therefore displacement, slope, bending moment and shear force are common at the radius $r=R_{f}$. The complete solution for the displacement on the surface of the constant thickness plate is now known and the implementation is 
described in further detail in section 4 .

$$
w(r, \theta, \omega)=\left(c_{1} J_{n}(\beta r)+c_{2} Y_{n}(\beta r)+c_{3} I_{n}(\beta r)+c_{4} K_{n}(\beta r)\right) e^{\mathrm{i} n \theta} e^{-\mathrm{i} \omega t} .
$$

\subsection{Circular tapered plate of specified profile}

In order to solve the equation of motion for the wedge-like section in Fig. 2, we consider the profile to follow $h(r)=\epsilon r^{m}$ where $\epsilon$ and $m$ are positive constants. In this case, the solution is complicated by the fact that the bending stiffness

alters as a function of the radius. The equation of motion for this case is given by Harris [11],

$$
\rho_{w} h_{w} \frac{\partial^{2} w_{w}(r, \theta, t)}{\partial t^{2}}=\left(1-v_{w}\right) \diamond^{4}\left\{D_{w}, w_{w}(r, \theta, t)\right\}-\nabla^{2}\left[D_{w} \nabla^{2} w_{w}(r, \theta, t)\right]
$$

where $\diamond^{4}\{D, w\}$ is the bilinear operator in polar coordinates (it is assumed that the bending stiffness is not a function of $\theta$ ).

$$
\diamond^{4}\{D, w\}=\frac{\partial^{2} D}{\partial r^{2}}\left(\frac{1}{r} \frac{\partial w}{\partial r}+\frac{1}{r^{2}} \frac{\partial^{2} w}{\partial \theta^{2}}\right)+\frac{\partial^{2} w}{\partial r^{2}}\left(\frac{1}{r} \frac{\partial D}{\partial r}\right)
$$

To complete the equation of motion, requires the differentiation of both $D$ and $w$ with respect to $r$.

$$
\nabla^{2}\left[D \nabla^{2} w\right]=\frac{\partial}{\partial r}\left[\frac{\partial}{\partial r}\left[D \nabla^{2} w\right]\right]+\frac{1}{r} \frac{\partial}{\partial r}\left[D \nabla^{2} w\right]+\frac{D}{r^{2}} \frac{\partial^{2}}{\partial \theta^{2}} \nabla^{2} w
$$

where after expansion of these terms, it may be shown that the full equation of motion can be written [1][12], 


$$
\begin{aligned}
& \frac{\partial^{4} w_{w}}{\partial r^{4}}\left[D_{w}\right]+\frac{\partial^{3} w_{w}}{\partial r^{3}}\left(\frac{2}{r}\left[D_{w}+r \frac{\partial D_{w}}{\partial r}\right]\right)+\frac{\partial^{2} w_{w}}{\partial r^{2}}\left(\frac{\partial^{2} D_{w}}{\partial r^{2}}+\frac{\partial D_{w}}{\partial r}\left[\frac{2}{r}+\frac{\nu}{r}\right]+D_{w}\left[-\frac{1}{r^{2}}\right]\right) \\
+ & \frac{\partial w_{w}}{\partial r}\left(\frac{\nu}{r} \frac{\partial^{2} D_{w}}{\partial r^{2}}-\frac{1}{r^{2}} \frac{\partial D_{w}}{\partial r}+D_{w}\left[\frac{1}{r^{3}}\right]\right)+\frac{\partial^{2} w_{w}}{\partial \theta^{2}}\left(\frac{\nu}{r^{2}} \frac{\partial^{2} D_{w}}{\partial r^{2}}+\frac{\partial D_{w}}{\partial r}\left[-\frac{3}{r^{3}}\right]+D_{w}\left[\frac{4}{r^{4}}\right]\right) \\
+ & \frac{\partial^{4} w_{w}}{\partial \theta^{4}}\left(\frac{D_{w}}{r^{4}}\right)+\frac{\partial^{4} w_{w}}{\partial r^{2} \partial \theta^{2}}\left(\frac{2 D_{w}}{r^{2}}\right)+\frac{\partial^{3} w_{w}}{\partial r \partial \theta^{2}}\left(-\frac{2 D_{w}}{r^{3}}+\frac{2}{r^{2}} \frac{\partial D_{w}}{\partial r}\right)+\rho_{w} h_{w} \frac{\partial^{2} w_{w}}{\partial t^{2}}=0 .
\end{aligned}
$$

Defining $\Gamma=E_{w} \epsilon^{3} / 12\left(1-\nu_{w}^{2}\right)$ allows the bending stiffness and derivatives to be written as,

$$
D_{w}=\Gamma r^{3 m}, \frac{\partial D_{w}}{\partial r}=3 m \Gamma r^{3 m-1}, \frac{\partial^{2} D_{w}}{\partial r^{2}}=3 m(3 m-1) \Gamma r^{3 m-2} .
$$

Substitution of these into the equation of motion and rearranging yields,

$$
\begin{aligned}
& r^{4} \frac{\partial^{4} w_{w}}{\partial r^{4}}+r^{3} \frac{\partial^{3} w_{w}}{\partial r^{3}}(2+6 m)+r^{2} \frac{\partial^{2} w_{w}}{\partial r^{2}}\left(3 m(3 m+1+\nu)-\left(1+2 n^{2}\right)\right) \\
+ & r \frac{\partial w_{w}}{\partial r}\left(3 m(3 m-1) \nu-3 m\left(1+2 n^{2}\right)+\left(1+2 n^{2}\right)\right) \\
+ & w_{w}\left(3 m\left(3 n^{2}-[3 m-1] \nu n^{2}\right)+n^{2}\left(n^{2}-4\right)\right)-\frac{12 \rho_{w} \omega^{2}\left(1-\nu^{2}\right)}{E_{w} \epsilon^{2}} w_{w} r^{4-2 m}
\end{aligned}
$$

As was pointed out by Conway [10], the only case where the last term in Eq. (15) becomes independent of $r$ is when the profile is given by $m=2$, which corresponds to the near zero free edge reflection coefficient in the framework of geometrical acoustics approximation [8]. Then the equation becomes homogeneous and an analytical solution is available for the case where $n=0$. To remove the radius in each of the differentials, a change of variable is employed to $z$ by assuming that $r=e^{z}$. Then the equation of motion can be written,

$$
\begin{aligned}
\frac{\partial^{4} w_{w}}{\partial z^{4}}+\frac{\partial^{3} w_{w}}{\partial z^{3}}(8) & +\frac{\partial^{2} w_{w}}{\partial z^{2}}\left(10+6 \nu-2 n^{2}\right)+\frac{\partial w_{w}}{\partial z}\left(-24+24 \nu-8 n^{2}\right) \\
& +w_{w}\left(n^{2}\left[n^{2}-30 \nu+14\right]-K\right)=0
\end{aligned}
$$

where $K=\frac{12 \rho_{w} \omega^{2}\left(1-\nu^{2}\right)}{E_{w} \epsilon^{2}}$. The solution to this differential equation is found by 
substituting $w(z)=e^{\lambda z}$ and finding the roots of the subsequent characteristic quartic equation. Conway provides the four solutions for $n=0$ as,

$$
\lambda_{1,2,3,4}=-2 \pm \sqrt{7-3 \nu \pm \sqrt{9\left(1-\nu^{2}\right)+K}}
$$

where the radial displacement is then given with reference to four constants.

$$
w_{w}(r, \theta, \omega)=c_{9} r^{\lambda_{1}}+c_{10} r^{\lambda_{2}}+c_{11} r^{\lambda_{3}}+c_{12} r^{\lambda_{4}} .
$$

For the case where $n \neq 0$, the roots in this paper are determined numerically using Lodovico Ferrari's method for quartic equations. For a wedge which includes a truncation radius, we then have four individual constants to determine.

The displacement of the plate and wedge surfaces are now to be found due to harmonic forcing. It is also useful to express the results as a function of the surface velocity, found using $\dot{w}(\omega) / p_{0}=\mathrm{i} \omega w(\omega) / p_{0}$ where the dot represents a differentiation with respect to time.

\section{Implementation to determine the vibration amplitude}

For the composite model comprising two constant thickness plates and one wedge-like section, we have twelve constants to determine. Therefore, twelve boundary conditions are required to determine the forced vibration case. This section provides the details of these conditions.

The solution method utilises three matrices; the matrix $\mathbf{C}$ is a twelve by one containing the constants $c_{1}$ to $c_{12}$. We then introduce two further matrices, so that $\mathbf{W}=\mathbf{X C}$, where $\mathbf{W}$ is a twelve by one in size containing the specific 
boundary conditions (e.g. bending moment is zero at $r=R_{t}$ ) and $\mathbf{X}$ is a twelve by twelve containing the specific equations from sections 3.1 and 3.2.

The boundary conditions applied are provided in tables 1 and 2, where the only non-zero element of $\mathbf{W}$ is shown to be the prescribed force $p$ at $r=R_{f}$ applied as an impulse $p\left(R_{f}\right)=\delta_{f}$ (when Fourier transforms are taken, this becomes a unity term). The full non-zero terms of the matrices are provided for completeness in the appendix, after Fourier transforms have been applied.

For a given angular order and frequency, the matrices are populated and numerically solved to find the twelve constants. Once these are known, it is possible to determine the displacement at a given radius $R_{m}$ by using Eq. (9).

In this paper, the results are shown as cross point mobilities $\dot{w}\left(R_{m}, n, \omega\right) / p\left(R_{f}\right)$, defined as the velocity of the plate surface at a radial position $R_{m}$ when a force is applied at a radial position $R_{f}$. The amplitude of this response is shown on a decibel scale $(\dot{w} / p) \mathrm{dB}$ which has been non-dimensionalised with a reference value of $1 \mathrm{~m} / \mathrm{s} / \mathrm{N}$.

\subsection{Plate dimensions and properties}

The results presented in this paper are predictions based on the vibration of a plate made of mild steel. The dimensions of the plates are provided in table 3 with notations for the dimensions as shown in Fig. 2. 


\subsection{Composite damping applied to the wedge-like section}

The material damping in the plate is a constant value derived from information provided in the literature. However, once an additional layer of damping material is applied to the plate, the overall damping changes. The details of the three damping methods used in this paper are provided in this section.

The plate material is assumed to be mild steel with an inherent damping value of $\eta=0.006[3]$ (which also applies to the wedge-like section if no additional damping material is applied). Any additional damping is provided through the use of a viscoelastic material (the subscript $d$ applies) with the following properties; The Young's modulus is $E_{d}=1 \times 10^{7} \mathrm{~Pa}$ and damping loss factor $\eta_{d}=0.2$. For the case where the damping is applied as a thin layer, it is assumed that the compressed layer has a thickness of $h_{d}=0.3 \mathrm{~mm}$, therefore the extensional stiffness of the damping layer is significantly less than the wedge or plate $(3 \mathrm{kN} / \mathrm{m}$ compared to $950 \mathrm{MN} / \mathrm{m})$. It is assumed that the mass of the damping layer is small in comparison to the mass of the wedge, which is reasonable given the density of most damping materials is significantly less than the density of mild steel. This paper uses a simple approximation of the composite damping in a plate of variable thickness, however the equations for the loss factor utilising thick viscoelastic layers which are also stiff in comparison to the base plate have been examined in [5], where the difference is only important at the very tip of the power-law. When considering the case of a constrained layer damping treatment, these additional terms are required over a wider range and have been used in this paper.

As the thickness of the wedge-like section changes with the radial position, the 
overall composite damping will also be a function of the radius. It would be possible to accurately model this change using a large number of thin vertical slices of plate, each of a slightly different height and damping, however, this would require a large number of numerical matrices. As a simplification, the equivalent composite damping for a wedge with a thin damping layer is determined based on the integrated reflection coefficient for a beam of quadratic power-law profile found through the application of geometrical acoustics approximation.

This equivalent loss factor is determined in two steps. Initially, the local composite loss factor is calculated for all radial positions on the wedge, using the work provided in Ross, Kerwin and Ungar [3]. For the case of a single layer of viscoelastic material applied to an isotropic, constant thickness elastic plate, the form given by Oberst (see also O'Boy et al. [7]) is utilised, where the composite damping for a given height of the wedge is given by,

$$
\eta_{\text {comp }}(r)=\frac{\eta_{D} \frac{E_{D} h_{D}}{E_{w} h_{w}}\left[4\left(\frac{h_{D}}{h_{w}}\right)^{2}+6\left(\frac{h_{D}}{h_{w}}\right)+3\right]}{1+\frac{E_{D} h_{D}}{E_{w} h_{w}}\left[4\left(\frac{h_{D}}{h_{w}}\right)^{2}+6\left(\frac{h_{D}}{h_{w}}\right)+5\right]} .
$$

It can be seen that the optimum damping is highly dependent on the extensional stiffness of the two materials. If the extensional stiffness of the damping layer is too high, or comparable to the base layer, then the assumptions in Eq. (19) break down. In addition, the profile of the wedge-like section will not follow a quadratic power-law design. The local composite damping for a single layer of damping material is shown in Fig. 3. The application of Eq. (19) assumes that the plate is a constant thickness, therefore the second step in deriving the equivalent loss factor for a damped wedge is to apply this method 
for many discrete steps of thickness.

In order that the numerical model is not split into a significantly large number of radial slices (each of slightly differing thickness), we require an equivalent single loss factor for the wedge without any applied damping layer which provides the same reflection coefficient as one with a damping layer. Krylov has determined the reflection coefficient for a flexural wave travelling into a 1D wedge material which reflects off a truncation using geometrical acoustics approximation. Although this is a 1D analysis, it will be used in this paper to determine the equivalent loss factor which is then substituted into the equations of motion for a radial wedge (the distance will be taken as a radial position).

The reflection coefficient $R_{0}$ for a flexural wave travelling into and out of a plain quadratic power-law wedge can be written [4],

$$
R_{0}=\exp \left\{-2 \frac{12^{1 / 4} \omega^{1 / 2}\left[\rho\left(1-\nu^{2}\right)\right]^{1 / 4}}{4 \epsilon^{1 / 2} E^{1 / 4}} \eta \int_{R_{t}}^{R_{i}} \frac{1}{r} \mathrm{~d} r\right\}
$$

where it is assumed that the material is isotropic with constant damping loss factor $\eta$.

When a thin damping layer is applied to the surface of the wedge, the local composite loss factor then changes with distance into the wedge, rising significantly close to the truncation point. The integration in Eq. (20) must then also take into account this variation inside the integral.

$$
R_{0}=\exp \left\{-2 \frac{12^{1 / 4} \omega^{1 / 2}\left[\rho\left(1-\nu^{2}\right)\right]^{1 / 4}}{4 \epsilon^{1 / 2} E^{1 / 4}} \int_{R_{t}}^{R_{i}} \frac{1}{r} \eta_{\mathrm{comp}}(r) \mathrm{d} r\right\}
$$

The expression for the local composite damping, Eq. (19), is substituted into Eq. (21) and the evaluation of the integral is completed numerically. If we 
assume that the reflection coefficients should be identical, we can determine a single equivalent loss factor which does not vary with the radial position, for a material which is isotropic but with the same reflection coefficient as one which is damped.

$$
\eta_{\text {equiv }}=\int_{R_{t}}^{R_{i}} \frac{1}{r} \eta_{\text {comp }}(r) \mathrm{d} r / \int_{R_{t}}^{R_{i}} \frac{1}{r} \mathrm{~d} r \text {. }
$$

Through the completion of Eq. (22), the equivalent composite damping for the wedge with a thin damping layer is determined as $\eta=0.015$. Modifying the thickness of the compressed damping layers yields the equivalent damping loss factors given in Table 4. Although this analysis for the composite damping is based on the equations of viscoelastic damping in constant thickness beams, by integrating the reflection coefficient for a large number of radial positions, each taking into account the thickness of damping material, base plate and their properties, we are able to obtain an estimate of the overall damping in the tapering wedge section for a flexural wave travelling through the section. The accuracy of this estimated average loss factor depends on the assumption that the damping of any point in the tapering section can be represented by a thin control volume of constant thickness damping film on a constant thickness base plate, therefore the change in thickness of the profile should be small (the assumptions would likely lead to larger errors for large power-laws).

An alternative damping method to be considered is the case of constrained layer damping, where the volume left by the machining of the wedge shape is filled with a depth of viscoelastic material and then a thin layer of mild steel placed on top, see Fig. 3. The main advantage of this method is that the external surface of the plate is smooth and flat. However, the equations 
in this case are slightly more complicated and are provided in Ross, Kerwin and Ungar (see Eq. (20) of [3]). These equations are used in this paper to estimate the local composite loss factor for a thick viscoelastic layer which is constrained.

The constraining layer (denoted with the subscript $c$ ) is assumed to be of the same material as the wedge, with a thickness $h_{c}=0.1 \mathrm{~mm}$. The composite damping of the plate using this constrained layer method compared with the thin layer is shown in Fig. 4. The constrained layer provides a greater loss factor at an earlier point on the wedge, except at the point closest to the truncation radius. At this point the constraining layer is thicker than the wedge and begins to dominate the equation.

It is also clear that it is only really useful to damp the final $4 \mathrm{~cm}$ of the wedge shaped section, otherwise the properties of the wedge material dominate. The effectiveness of damping the thin wedge material is shown. Therefore, for optimum damping, the plate must taper to as thin as possible in as short a distance as possible, but without creating such a large impedance change that waves travelling into the wedge are reflected.

The average composite damping utilised in the results section for constrained layer damped wedge-like section is $\eta=0.0286$ for frequencies less than $500 \mathrm{~Hz}$ rising rapidly to approximately $\eta=0.0798$ above this. This could be increased by removing the constraining layer closer to the truncation point, where it appears to be less efficient, reducing the local composite loss factor. 


\section{Results}

In this section, the results for the vibration of the different plates are provided. As shown in the mathematical derivation of the numerical model, the vibration of the plate surface can be presented as functions of the amplitude of displacement of certain modes or as functions of spatial coordinates. In this paper, we present selected results for both, although the application of damped wedge-like holes is not restricted to any single mode of vibration.

In order to obtain confidence in the predictions provided by the numerical model, comparisons are made against experimental measurements on an annulus machined from mild steel which has a lower overall diameter than the results presented in the rest of the paper. One key advantage is that the resonances are more spread out in frequency, such that an accurate comparison can be made.

Once the predictions for the model are validated, the mode shapes for different values of the angular order are then presented, then the Fourier inversion is carried out from $n$ to $\theta$ and results for the vibration amplitudes of the composite plate with different damping methods are then shown as cross point mobility functions where the forcing and measurement locations are assumed to occur at $\theta=0 \mathrm{rad}$.

\subsection{Validation of numerical models against experimental measurements}

The comparison between the numerical predictions and experimental measurements is considered separately from the results in the rest of the paper in 
order to ensure that any predictions made have sufficient accuracy in terms of the frequency location of any resonances and their amplitudes. Two plates were machined from the same piece of cold rolled mild steel with the same material properties as shown in Table 3 . In order to be able to identify individual resonances more clearly, the overall diameter of the plate is reduced to $0.5 \mathrm{~m}$, with a thickness of $5.07 \mathrm{~mm}$. The first plate is a simple annulus with a free edge at a diameter of $0.20 \mathrm{~m}$ and no damping applied. The second is an identical plate, however, at the diameter of $0.20 \mathrm{~m}$, a wedge indentation is machined following a quadratic power-law profile, which terminates at a truncation diameter of $10 \mathrm{~mm}$. The power-law profile is machined in 50 discrete step positions, although it will be assumed that this is approximately similar to a continuous profile. A thin elastic damping material was placed on the end of the wedge, with parameters approximately matching the numerical predictions in this paper, however, due to the difficulty in determining the accurate local loss factor (as the damping tape adhesive varied in different positions on the discrete surface) the comparisons of amplitude variation will be minimised.

The driving point mobility was measured at an arbitrary diameter of $0.35 \mathrm{~m}$ using a standard Bruel and Kjaer accelerometer (series 4371) and a broadband electromagnetic shaker through a Bruel and Kjaer force transducer (series 8200 ). The acceleration, recorded in $8 \mathrm{~Hz}$ increments up to a frequency of $6.4 \mathrm{kHz}$ was subsequently converted into velocity during post processing. The experimental measurements were carried out at the Noise and Vibration Laboratory of Loughborough University with the assistance of E. P. Bowyer, using a supporting rig designed to replicate free boundary conditions on the outer surfaces. 
The numerical calculations also used a sampling interval of $8 \mathrm{~Hz}$, with all other parameters replicated as in the experimental measurements. The comparison between the numerical predictions of an annular plate and the experimental measurements is shown in Fig. 5. The location of each of the resonances and anti-resonances is broadly well matched, for example, the resonances at 0.59 , $2.22,2.78,3.38,3.98$ and $4.7 \mathrm{kHz}$ have errors in the peak frequency location of $1.7 \%, 2.7 \%, 1.4 \%, 0.3 \%, 1.3 \%$ and $0.2 \%$ respectively.

The comparison of the predicted amplitudes against experimental measurements is less accurate at lower frequencies, most likely due to low frequency damping thorough the boundary conditions on the edges of the plate. At frequencies of $0.59,2.22,2.78,3.38$ and $4.7 \mathrm{kHz}$ the difference in peak amplitudes are $6 \mathrm{~dB}, 10 \mathrm{~dB}, 1 \mathrm{~dB}, 0.5 \mathrm{~dB}$ and $0.1 \mathrm{~dB}$ respectively. We may therefore conclude that the numerical model and solution method provides sufficient accuracy once above an excitation frequency of $2.5 \mathrm{kHz}$.

The experimental measurements are then repeated on a plate which has a central indentation profile following a quadratic power-law design. This is covered with a layer of damping material. The results are shown in Fig. 6, where it may be seen again that the location of the frequencies are broadly correct. In this case, the low frequency amplitudes are better matched however, the measurements show much more variable damping at higher frequencies than the predictions indicate. These variations are likely to be due to the inconsistent local adhesion of the damping tape to the metal surface, even after cleaning with alcohol solution. Further comparison and practical implementation of this damping method will require a more efficient attachment method for the damping material to the metal plate. 
The model does agree with the experimental measurements sufficiently to have confidence in the predictions though. As an example, at the resonance at $2.75 \mathrm{kHz}$, the error in the frequency location is only $1.1 \%$, with an error in amplitude of only $1 \mathrm{~dB}$. The numerical model is now used to calculate the cross point mobility of the composite plate with dimensions as shown in Table 3.

\subsection{Composite plate resonances as functions of angular order}

The cross mobility measurements of the composite plate, with a constant thickness plate of outer radius $r=R_{o}$ and inner radius $R_{i}$ and a wedge attachment terminating at a radius $R_{t}$ are shown in Fig. 7 for three different angular orders. In the frequency range $0-5 \mathrm{kHz}$, there are only three resonances for each order, each representing an additional nodal diameter on the plate.

Examples of these response shapes are provided in Figs. 8(a)-(i), illustrated as displacements at a single time. The amplitude on the wedge close to the termination point is not shown, as it becomes large in comparison to that on the main plate surface (such that the nodal lines cannot be seen clearly), although these displacements are small in comparison to the plate thickness. The figures (a)-(c) refer to the $n=0$ mode, while (d)-(f) relate to $n=2$ and (g)-(i) refer to $n=4$. Without a truncation in the circular plate on the power-law profile, the assumptions in the differential equation leading to the equation of motion are violated, as the amplitude becomes far greater than the thickness in the immediate vicinity of $r=0$. However, as soon as a truncation is introduced, the amplitude, while still large, never becomes infinitely high. Although there will still be some errors in this model, it provides an effective 
and simple model for vibrations including quadratic tapered geometries. If the truncation position of the profile is further reduced (through a different machining technique to that described in this paper), it is likely that large displacement terms would be required in the equation of motion, or the use of the full equations of elasticity to provide an analysis in the immediate vicinity of $r=0$. In this paper, we assume that the displacement on any part of the plate or profiled surface is small in comparison to the plate thickness.

Analysis of the amplitude of displacement in each mode shows that it is possible to neglect values of angular order greater than $n=30$ in the Fourier inversion, as the cut-on frequencies extend higher than $10 \mathrm{kHz}$. Therefore the Fourier sum can be written as, $w(\theta)=\tilde{w}(n=0)+\left(\sum_{n=1}^{n=30} \tilde{w}(n)\left(e^{\mathrm{i} n \theta}+e^{-\mathrm{i} n \theta}\right)\right.$.

\subsection{Comparison of damped circular plate to damped composite structure}

The Fourier sum over angular order is now completed, with results presented at an angle $\theta=0 \mathrm{rad}$. The cross point mobility calculations for a solid constant thickness disc of radius $r=R_{o}$ are shown in Fig. 9(a), with a comparison to a disc which has a circular inner hole at $r=R_{i}$. The overall amplitude of the response from the small disc is between 5-10 dB higher as shown in Fig. 9(c) which provides further detail for the frequencies below $750 \mathrm{~Hz}$. Once above a frequency of $2.5 \mathrm{kHz}$, the resonances start to diverge from each other such that the peaks and troughs do not line up at the same frequency.

In the subsequent plot, Fig. 9(b), the lighter of these two discs (outer radius $R_{o}$ and inner radius $R_{i}$ ) is compared to a similar disc, with an additional profiled wedge section extending from $R_{i}$ to $R_{t}$. Two trends are apparent, 
that the overall amplitude response is similar to the solid disc, except at the lowest frequency where the amplitude is significantly higher, see the detailed low frequency region in Fig. 9(d).

It is therefore apparent that except at very low frequencies, the inclusion of a profiled wedge section does not alter the overall mobility response of a solid plate, only the location of those frequencies. Therefore the addition of a free wedge-like section can not be considered as a possible damping method for vibrating structures.

We now present results for two damping methodologies. In Fig. 10 a thin damping layer is applied to the whole of the constant thickness plate. This is likely to be the most common method of damping unwanted vibrations which lead either to fatigue or acoustic problems. However, it is also extremely inefficient.

For an excitation frequency below $500 \mathrm{~Hz}$, the additional damping layer provides little reduction in the amplitude. For a mid-frequency range, the reduction is only $2-2.5 \mathrm{~dB}$ and above $3 \mathrm{kHz}$, where the damping would be expected to have the most impact, the reduction is only $2.5-3 \mathrm{~dB}$. This matches the predictions by Ross, Kerwin and Ungar [3] in section 4.2, who showed that the extensional stiffness of the plate layer dominates that of the damping layer, resulting in a very low composite damping coefficient (in this case $\left.\eta=8.45 \times 10^{-3}\right)$. Even through adhering a damping layer to the whole surface and covering the whole plate with damping film, the effect is minimal. This contrasts with the enhanced amplitude reduction found by damping a profiled wedge section. In Fig. 11, the amplitude response of the same disc is compared with one where a profiled wedge-like section has been added (covered with the 
same thickness of damping film). The composite damping for the wedge is now a larger coefficient $\eta=1.51 \times 10^{-2}$, as the thickness of the damping layer is comparable to the thickness of the wedge close to the centre of the plate.

At the lowest frequencies, the amplitude response of the damped wedge plate is still much higher. However, as the frequency increases, the amplitude is reduced by between $7-14 \mathrm{~dB}$, with the largest reductions appearing at the higher frequency range. In this case, the volume of damping material is far smaller than that needed to cover the whole plate and yet the reductions are more significant.

A disadvantage of damping vibrations using small profiled sections is that the original plate is now not flat and smooth. A possible solution could be to utilise a constrained layer damping method, where the constraining layer is of the same material as the plate and is flush with the top surface. An added advantage would be an expected increase in shear damping, especially at higher frequencies.

This comparison is shown in Fig. 12 where the composite damping is now a higher $\eta=7.98 \times 10^{-2}$ for the majority of the frequency range. It can be shown that the effect of this damping method is to achieve an additional 1$3 \mathrm{~dB}$ reduction over the thin damping layer. This is highly dependent on the frequency (at low frequencies the overall reduction is between $3-8 \mathrm{~dB}$, whereas at the highest frequencies a $17 \mathrm{~dB}$ overall reduction is possible).

Further work is required to compare these predictions to those which are found when the numerical terms incorporating rotary inertia and shear deformation are included in the plate equations. Also, comparisons with experimental measurements will verify the predicted large $16 \mathrm{~dB}$ increase in amplitude at the 
first mode of vibration.

The predictions have shown the benefit of including a damped wedge-like structure to minimise amplitude of vibration. A summary table of the amplitude reductions is provided in table 5 . The reference plate is the circular plate with outer and inner dimensions $R_{o}$ and $R_{i}$ respectively. The subsequent reductions and increases are then shown for the alternative plates.

This paper has shown that lightly damped tapered wedge indentations can provide a greater reduction in peak vibration amplitude than can be found by covering the whole plate with damping tape. We now provide additional results for the case where the thickness of the damping film attached to the wedge is varied, by the number of layers applied. The comparison of the peak vibration amplitude of the cylindrical plate incorporating a damped wedge indentation is shown in Fig. 13 where the number of damping layers applied to the wedge are varied, implemented through a variable equivalent loss factor as discussed in section 4.2 .

The upper frequency range of the results are shown, where it may be seen that the mobility response can be separated into longitudinal modes of vibration and across width modes. The damping applied to the wedge only substantially affects the longitudinal modes and therefore the resonances at $2.94 \mathrm{kHz}$ and $3.48 \mathrm{kHz}$ do not show any appreciable difference in amplitude with an increase in the number of applied damping layers, as expected.

Calculations carried out according to the above mentioned approach show that the amplitude of the longitudinal modes varies substantially with an increase in the number of damping layers, decreasing by 4, 5.5, 7.5 and $7.75 \mathrm{~dB}$ for two, three, six and eight layers respectively at a frequency of $2.55 \mathrm{kHz}$. At 
the higher frequency of $3.74 \mathrm{kHz}$, these reductions are 2.6, 4.0, 5.5, $6.2 \mathrm{~dB}$ respectively. The advantage of increasing the number of layers is clear through the amplitude reductions, at the expense of an increase in the mass of the damping layers applied. Of interest is that, according to the above calculations, the plate with approximately eight layers of damping tape reduces the overall amplitude by slightly more than the reduction found through the use of the constrained layer method. This is due to a greater local loss factor closer to the tip of the wedge indentation, where the constraining layer is not as efficient and actually increases the extensional stiffness significantly. However, the clear potential disadvantage is that the outer plate surface is not flat and smooth, which may be important for different applications.

\section{Conclusions}

Mobilities for a circular plate with a tapered central hole of power-law profile have been numerically calculated using the exact solution of the corresponding flexural wave equation that exists for $m=2$. Note that this value of $m$ belongs to the range $m \geq 2$ that defines zero reflection of quasi-plane waves from a tapered hole in geometrical acoustics approximation.

Two cases of added damping in the central hole area have been considered: a thin absorbing layer and a constrained layer. Cross point mobilities have been calculated for both these cases in a situation when the points of excitation and observation are characterised by the same polar angle. In a particular case of equal polar radii these give results for point mobilities.

The obtained results for point and cross mobilities show a substantial sup- 
pression of resonant peaks (up to $17 \mathrm{~dB}$ ), in comparison with the cases of a plate with an uncovered hole of the same power-law profile and of a reference circular plate of constant thickness covered or uncovered by a thin absorbing layer. Apparently, this suppression is associated with the near zero reflection coefficient caused by a tapered hole with $m=2$.

Although the geometry of a circular plate with a tapered hole of power-law profile considered in this paper is highly symmetrical and applicable to a restricted number of practical applications, the obtained numerical results for mobilities are important as they give a clear idea of what degree of damping can be expected from two-dimensional power-law profile holes. It is expected that the same order of damping will also take place in a variety of practical situations, e.g. for rectangular plates or other structures with arbitrary locations of tapered holes, etc. Further theoretical and experimental research is needed to examine these expectations.

\section{Acknowledgements}

The research reported here has been supported by EPSRC grant EP/F009232/1. 


\section{Appendix: Terms for a circular plate with a profiled wedge addition}

The matrices relating to the numerical model of the composite plate (two constant thickness circular plates with a profiled wedge section at the centre) utilise a notation $\mathbf{W}=\mathbf{X} \mathbf{C}$. The full terms of these matrices are provided in this section for completeness (the expression $e^{\mathrm{i} n \theta} e^{-\mathrm{i} \omega t}$ has been omitted).

The matrix $\mathbf{C}$ of constants is twelve rows by one column. The first four relate to the first constant thickness plate, the next four relate to the second constant thickness plate and the final four relate to the wedge.

$$
\mathbf{C}=\left(c_{1}, c_{2}, c_{3}, c_{4}, c_{5}, c_{6}, c_{7}, c_{8}, c_{9}, c_{10}, c_{11}, c_{12}\right)^{T}
$$

The matrix $\mathbf{W}$ contains the results of the boundary conditions, whose only non-zero term is $W[6]=1$ as the prescribed shear force applied as an impulse at the join between the two constant thickness plates $r=R_{f}$.

The matrix $\mathbf{X}$ contains the applied boundary conditions, a twelve by twelve in size. The non-zero entry for $J_{n}\left(\beta R_{o}\right)$ in the first boundary condition is given by,

$$
X[1,1]=\left(\frac{n(n-1)(1-\nu)}{R_{o}^{2}}-\beta^{2}\right) J_{n}\left(\beta R_{o}\right)+\left(\frac{\beta}{R_{o}}(1-\nu)\right) J_{n+1}\left(\beta R_{o}\right),
$$

where the subsequent terms for $\mathrm{X}[1,2], \mathrm{X}[1,3]$ and $\mathrm{X}[1,4]$ can be found by substituting $Y_{n}, I_{n}, K_{n}$ respectively instead of $J_{n}$.

Similarly the second boundary condition has an entry, 


$$
\begin{aligned}
X[2,1]= & \left(\frac{n^{2}(n-2)}{R_{o}^{3}}-\frac{\beta^{2} n}{R_{o}}+\frac{(3-\nu) n^{2}}{R_{o}^{3}}-\frac{(2-\nu) n^{3}}{R_{o}^{3}}\right) J_{n}\left(\beta R_{o}\right)+ \\
& \left(\beta^{3}+\frac{\beta(1-\nu) n^{2}}{R_{o}^{2}}\right) J_{n+1}\left(\beta R_{o}\right),
\end{aligned}
$$

The third to the sixth boundary conditions are applied at a radius $r=R_{f}$, the forcing location. The terms in $J_{n}$ are;

$$
\begin{aligned}
& X[3,1]=J_{n}\left(\beta R_{f}\right), \quad X[3,5]=-X[3,1] . \\
& X[4,1]=\left(\frac{n}{R_{f}}\right) J_{n}\left(\beta R_{f}\right)-\beta J_{n+1}\left(\beta R_{f}\right), \quad X[4,5]=-X[4,1] . \\
& X[5,1]=\left(\frac{n(n-1)(1-\nu)}{R_{f}^{2}}-\beta^{2}\right) J_{n}\left(\beta R_{f}\right)+\left(\frac{\beta}{R_{f}}(1-\nu)\right) J_{n+1}\left(\beta R_{f}\right), \quad X[5,5]=-X[5,1] . \\
& X[6,1]=\left(\frac{n^{2}(n-2)}{R_{f}^{3}}-\frac{\beta^{2} n}{R_{f}}+\frac{(3-\nu) n^{2}}{R_{f}^{3}}-\frac{(2-\nu) n^{3}}{R_{f}^{3}}\right) J_{n}\left(\beta R_{f}\right)+ \\
& \left(\beta^{3}+\frac{\beta(1-\nu) n^{2}}{R_{f}^{2}}\right) J_{n+1}\left(\beta R_{f}\right), \quad X[6,5]=-X[6,1] .
\end{aligned}
$$

The matrix terms X[3,2], X[3,3], X[3,4] and X[3,6], X[3,7], X[3,8] are determined by substituting the alternate Bessel's functions to $J_{n}$.

The final six boundary conditions relate to the join between the second constant thickness plate and the profiled wedge. The boundary conditions applied at this radius, $r=R_{i}$ are,

$$
\begin{gathered}
X[7,1]=J_{n}\left(\beta R_{i}\right) . \\
X[8,1]=\left(\frac{n}{R_{i}}\right) J_{n}\left(\beta R_{i}\right)-\beta J_{n+1}\left(\beta R_{i}\right) . \\
X[9,1]=\left(\frac{n(n-1)(1-\nu)}{R_{i}^{2}}-\beta^{2}\right) J_{n}\left(\beta R_{i}\right)+\left(\frac{\beta}{R_{i}}(1-\nu)\right) J_{n+1}\left(\beta R_{i}\right) .
\end{gathered}
$$




$$
\begin{aligned}
& X[10,1]=\left(\frac{n^{2}(n-2)}{R_{i}^{3}}-\frac{\beta^{2} n}{R_{i}}+\frac{(3-\nu) n^{2}}{R_{i}^{3}}-\frac{(2-\nu) n^{3}}{R_{i}^{3}}\right) J_{n}\left(\beta R_{i}\right)+ \\
&\left(\beta^{3}+\frac{\beta(1-\nu) n^{2}}{R_{i}^{2}}\right) J_{n+1}\left(\beta R_{i}\right) . \\
& X[7,9]=-R_{i}^{\lambda_{1}}, X[7,10]=-R_{i}^{\lambda_{2}}, X[7,11]=-R_{i}^{\lambda_{3}}, X[7,12]=-R_{i}^{\lambda_{4}} . \\
& X[8,9]=-\lambda_{1} R_{i}^{\lambda_{1}-1}, X[8,10]=-\lambda_{2} R_{i}^{\lambda_{2}-1} \\
& X[8,11]=-\lambda_{3} R_{i}^{\lambda_{3}-1}, X[8,12]=-\lambda_{4} R_{i}^{\lambda_{4}-1} .
\end{aligned}
$$

At $r=R_{t}$, the truncation radius of the wedge, the final two boundary conditions reflect a free edge condition.

\section{References}

[1] S. P. Timoshenko, Theory of Plates and Shells, New York: McGraw-Hill, 1959.

[2] K. F. Graff, Wave Motion in Elastic Solids, Clarendon Press, Oxford, 1975.

[3] D. Ross, E. M. Kerwin, E. E. Ungar, Damping of plate flexural vibrations by means of viscoelastic laminae, in Structural Damping (Ruzicka, J. E., ed) 3 (1959) 44-87.

[4] V. V. Krylov, F. J. B. S. Tilman, Acoustic 'black holes' for flexural waves as effective vibration dampers, Journal of Sound and Vibration 274 (3-5) (2004) 605-619.

[5] V. V. Krylov, New type of vibration dampers utilising the effect of acoustic 'black holes', Acta Acustica united with Acustica 90 (5) (2004) 830-837.

[6] V. V. Krylov, R. E. T. B. Winward, Experimental investigation of the acoustic black hole effect for flexural waves in tapered plates, Journal of Sound and Vibration 300 (1) (2007) 43-49. 
[7] D. J. O'Boy, V. V. Krylov, V. Kralovic, Damping of flexural vibrations in rectangular plates using the acoustic black hole effect, Journal of Sound and Vibration 329 (22) (2010) 4672-4688.

[8] V. V. Krylov, Propagation of plate bending waves in the vicinity of oneand two-dimensional acoustic black holes, in: Proceedings of the International Conference on Computational Methods in Structural Dynamics and Earthquake Engineering, COMPDYN 2007, Crete, Greece, 13-26 June, 2007, (on CD).

[9] F. Gautier, J. Cuenca, V. V. Krylov, L. Simon, Experimental investigation of the acoustic black hole effect for vibration damping in elliptical plates (abstract), in: Second Acoustical Society of America and European Acoustics Association joint conference (Congrés Acoustics'08), Paris, 24-29 June, 2008.

[10] H. D. Conway, Some special solutions for the flexural vibrations of discs of varying thickness, Ing.-Arch. 26 (1958) 408-410.

[11] G. Z. Harris, The normal modes of a circular plate of variable thickness, Quarterly Journal of Mechanics and Applied Mathematics 21 (3) (1967) 331327.

[12] R. K. Jain, Vibrations of circular plates of variable thickness under an inplane force, Journal of Sound and Vibration 23 (4) (1972) 407-414.

[13] P. A. A. Laura, Static and dynamic behavior of circular plates of variable thickness elastically restrained along the edges, Journal of Sound and Vibration 52 (2) (1997) 243-251.

[14] U. S. Gupta, L. Lal, Buckling and vibrations of circular plates of variable thickness, Journal of Sound and Vibration 58 (4) (1978) 501-507.

[15] U. S. Gupta, L. Lal, Vibrations and buckling of parabolically tapered circular plates, Indian Journal of Pure and Applied Mathematics 10 (3) (1979) 347-356. 
[16] B. Singh, V. Saxena, Axisymmetric vibration of a circular plate with double linear variable thickness, Journal of Sound and Vibration 179 (5) (1995) 879897.

[17] B. Singh, V. Saxena, Axisymmetric vibration of a circular plate with exponential thickness variation, Journal of Sound and Vibration 192 (1) (1996) $35-42$.

[18] U. S. Gupta, A. H. Ansari, Free vibration of polar orthotropic circular plates of variable thickness with elastically restrained edge, Journal of Sound and Vibration 213 (3) (1998) 429-445.

[19] H. Rokni Damavandi Taher, M. Omidi, A. A. Zadpoor, A. A. Nikooyan, Free vibration of circular and annular plates with variable thickness and different combinations of boundary conditions, Journal of Sound and Vibration 296 (45) (2006) 1084-1092.

[20] I. Elishakoff, Axisymmetric vibration of inhomogeneous clamped circular plates: An unusual closed-form solution, Journal of Sound and Vibration 233 (4) (2000) $727-738$.

[21] J. S. Yang, The vibration of a circular plate with varying thickness, Journal of Sound and Vibration 165 (1) (1993) 178-184.

[22] X. Wang, J. Yang, J. Xiao, On free vibration analysis of circular annular plates with non-uniform thickness by the differential quadrature method, Journal of Sound and Vibration 184 (3) (1995) 547-551. 


\section{List of figure and table captions}

Fig. 1. A circular plate incorporating a profiled wedge-like section at the centre. A small element has been removed to illustrate the change in thickness with radial position.

Fig. 2. Cross section of the composite model, composed of two constant thickness plates and one tapered plate, all of circular shape. The point at which a force is applied and vibration displacement measured are also shown.

Fig. 3. Constrained layer damping; The wedge-like hole volume is filled with a viscoelastic damping layer and a thin material layer is placed on top, such that the overall plate surface is flat.

Fig. 4. Composite damping coefficient of the profiled wedge comparing a constrained layer damping solution with a thin layer of damping film of constant thickness. The wedge-like section radius extends from $r=R_{t}$ to $R_{i}$.

Fig. 5. Comparison of the predicted driving point mobility $\dot{w}\left(R_{m}, \theta=0, \omega\right) / p\left(R_{m}\right)$ for an annulus against experimental measurements.

Fig. 6. Comparison of the predicted driving point mobility $\dot{w}\left(R_{m}, \theta=0, \omega\right) / p\left(R_{m}\right)$ for an annulus with a damped quadratic power-law indentation machined into it, against experimental measurements. 
Fig. 7. Cross point mobility calculations of the composite plate, $\dot{w}\left(R_{m}, n, \omega\right) / p\left(R_{f}\right)$ for three angular orders $(n=0,2,4)$.

Fig. 8. Examples of the displacement response shapes at prescribed frequencies and angular orders; For a frequency (a) $f=0.22 \mathrm{kHz}$, (b) $f=1.85 \mathrm{kHz}$, (c) $f=3.74 \mathrm{kHz}$, (d) $f=0.49 \mathrm{kHz}$, (e) $f=1.20 \mathrm{kHz}$, (f) $f=2.20 \mathrm{kHz}$, (g) $f=0.98 \mathrm{kHz}$, (h) $f=1.90 \mathrm{kHz}$, (i) $f=3.10 \mathrm{kHz}$.

Fig. 9. Comparison of the cross point mobility calculations of three different plates after the Fourier sum over angular order has been completed, $\dot{w}\left(R_{m}, \theta=0, \omega\right) / p\left(R_{f}\right)$. Plates are a) a constant thickness plate from $r=R_{o}$ to $r=0$ against a constant thickness plate from $r=R_{o}$ to $r=R_{i}$; b) a constant thickness plate from $r=R_{o}$ to $r=R_{i}$ compared against a constant thickness plate from $r=R_{o}$ to $r=R_{i}$ with a profiled wedge section from $R_{i}$ to $R_{t}$. The low frequency regions of these two results are shown in detail in figures c) and d) respectively where the same legends apply.

Fig. 10. Comparison of the cross point mobility calculations of two different plates, $\dot{w}\left(R_{m}, \theta=0, \omega\right) / p\left(R_{f}\right)$. Plates are a constant thickness plate from $r=R_{o}$ to $r=R_{i}$ with only inherent metal damping against the same plate covered with a layer of damping film (the composite damping coefficient is $\eta=8.45 \times 10^{-3}$ ).

Fig. 11. Comparison of the cross point mobility calculations of two different plates, $\dot{w}\left(R_{m}, \theta=0, \omega\right) / p\left(R_{f}\right)$. Plates are a constant thickness plate from $r=R_{o}$ to $r=R_{i}$ with only inherent metal damping against the same plate with a profiled wedge section from $r=R_{i}$ to $R_{t}$ covered with a layer of damping film (the composite damping coefficient is $\eta=1.51 \times 10^{-2}$ ). 
Fig. 12. Comparison of the cross point mobility calculations of two different plates, $\dot{w}\left(R_{m}, \theta=0, \omega\right) / p\left(R_{f}\right)$. Plates are a constant thickness plate from $r=R_{o}$ to $r=R_{i}$ with a profiled wedge attachment from $r=R_{i}$ to $R_{t}$ compared with the same plate when the wedge-like hole is covered with a constrained layer damping solution (the composite damping coefficient is $\eta=7.98 \times 10^{-2}$ ).

Fig. 13. Comparison of the cross point mobility calculations of the cylindrical plate with a damped wedge profile, $\dot{w}\left(R_{m}, \theta=0, \omega\right) / p\left(R_{f}\right)$. The thickness of damping layer applied to the surface is varied such that the peak amplitude response is further reduced. 
Table 1

Boundary conditions applied to the outer constant thickness plate (1) and the inner constant thickness plate (2).

Table 2

Boundary conditions applied to the inner constant thickness plate and the wedgelike section.

Table 3

Parameters of the composite plate used in the numerical predictions.

Table 4

Equivalent loss factors for a wedge with a different number of layers of thin elastic damping tape applied to the surface.

Table 5

Summary of potential reductions in plate resonance using different damping methods. The reference calculation is the cross mobility response of a constant thickness plate of outer radius $r=R_{o}$ and inner radius $R_{i}$. The subsequent reductions in $\mathrm{dB}$ re $1 \mathrm{~m} / \mathrm{s} / N$ are given for the reference plate covered with damping film, the reference plate with an undamped and damped wedge section and finally with a constrained layer damping applied to the wedge section. 
List of figures, tables and captions

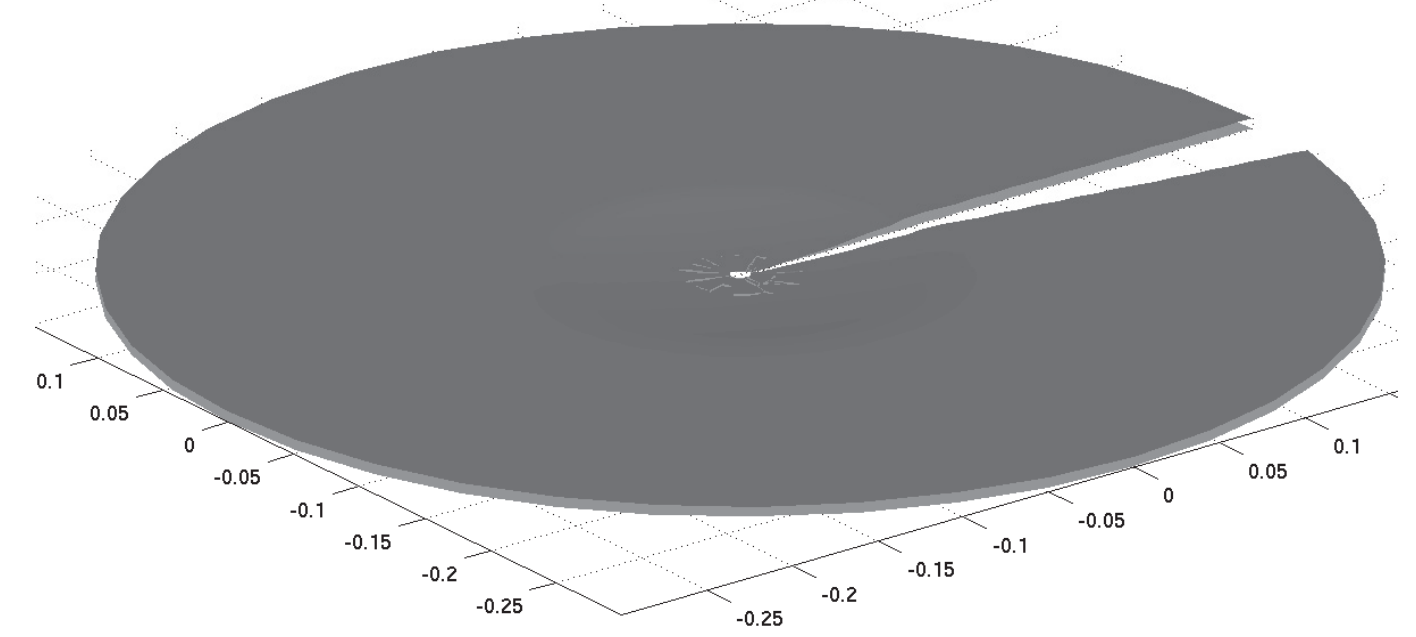

Fig. 1. A circular plate incorporating a profiled wedge-like section at the centre. A small element has been removed to illustrate the change in thickness with radial position. 


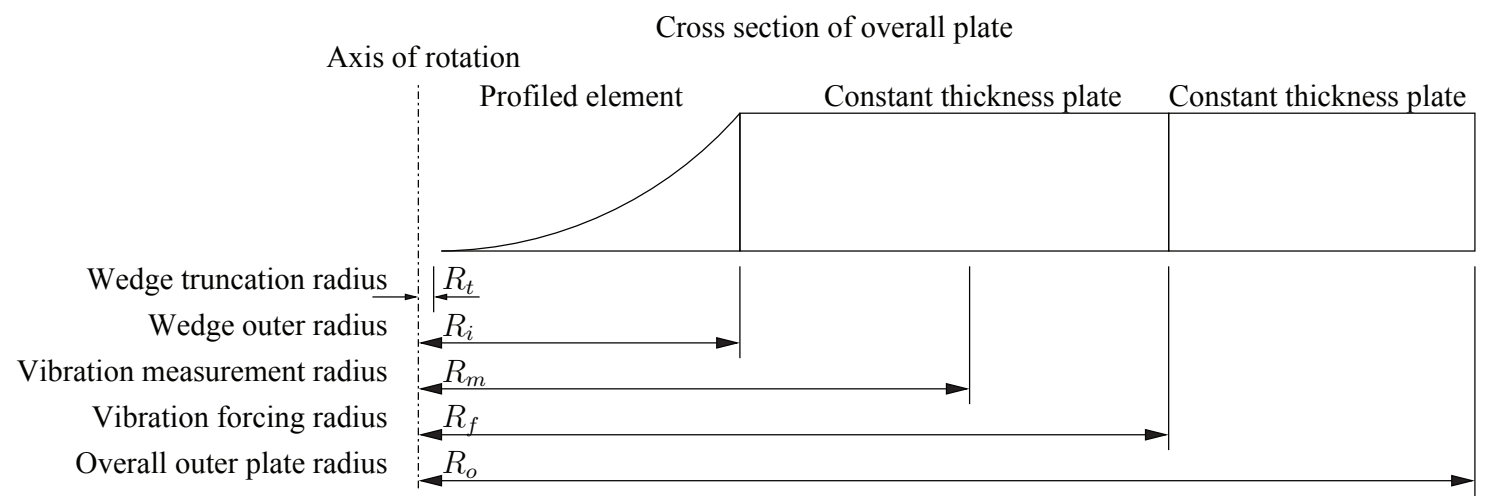

Fig. 2. Cross section of the composite model, composed of two constant thickness plates and one tapered plate, all of circular shape. The point at which a force is applied and vibration displacement measured are also shown. 


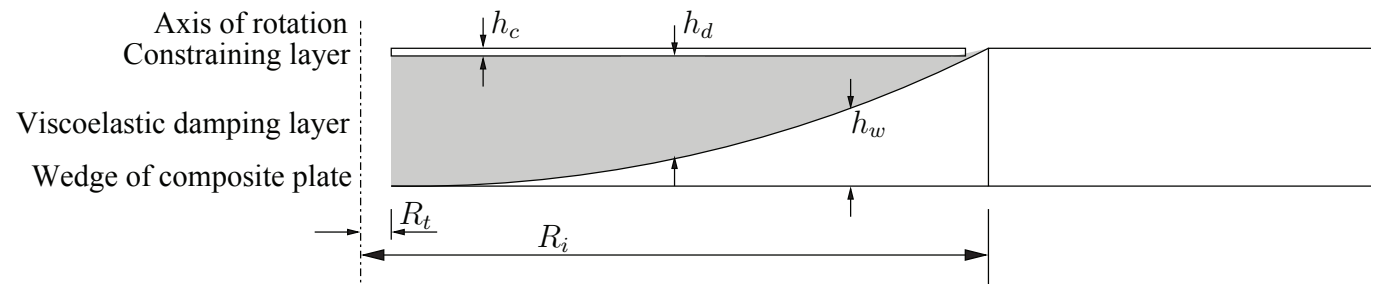

Fig. 3. Constrained layer damping; The wedge-like hole volume is filled with a viscoelastic damping layer and a thin material layer is placed on top, such that the overall plate surface is flat. 


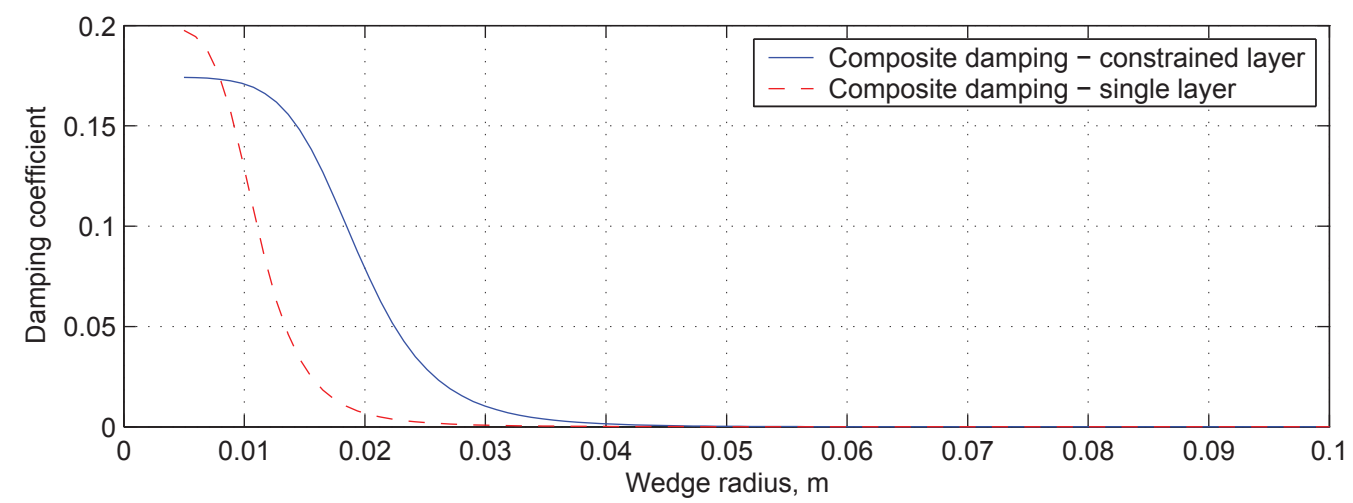

Fig. 4. Composite damping coefficient of the profiled wedge comparing a constrained layer damping solution with a thin layer of damping film of constant thickness. The wedge-like section radius extends from $r=R_{t}$ to $R_{i}$. 


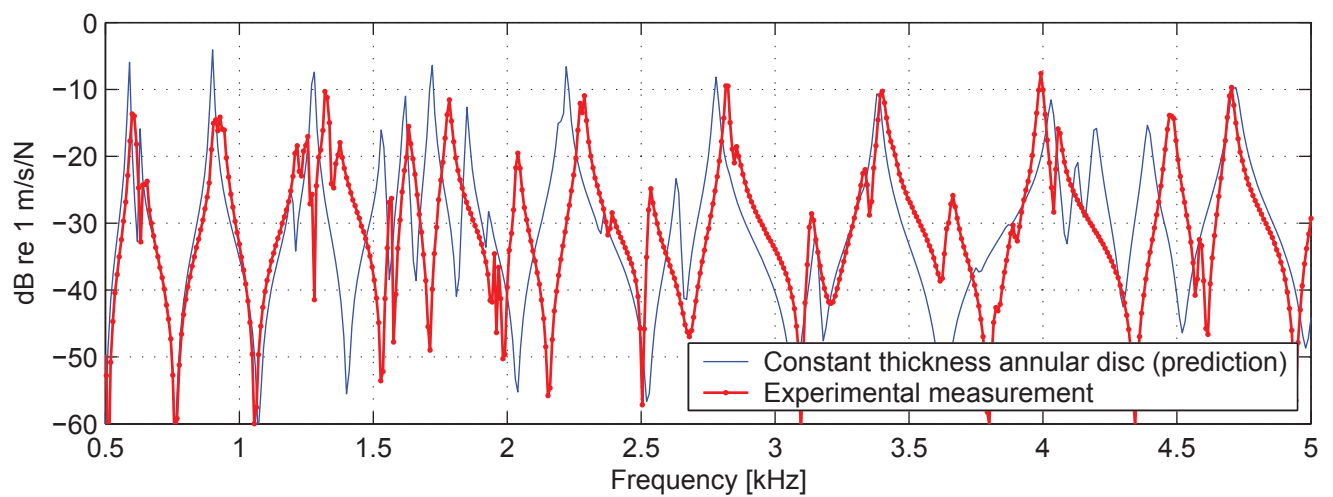

Fig. 5. Comparison of the predicted driving point mobility $\dot{w}\left(R_{m}, \theta=0, \omega\right) / p\left(R_{m}\right)$ for an annulus against experimental measurements. 


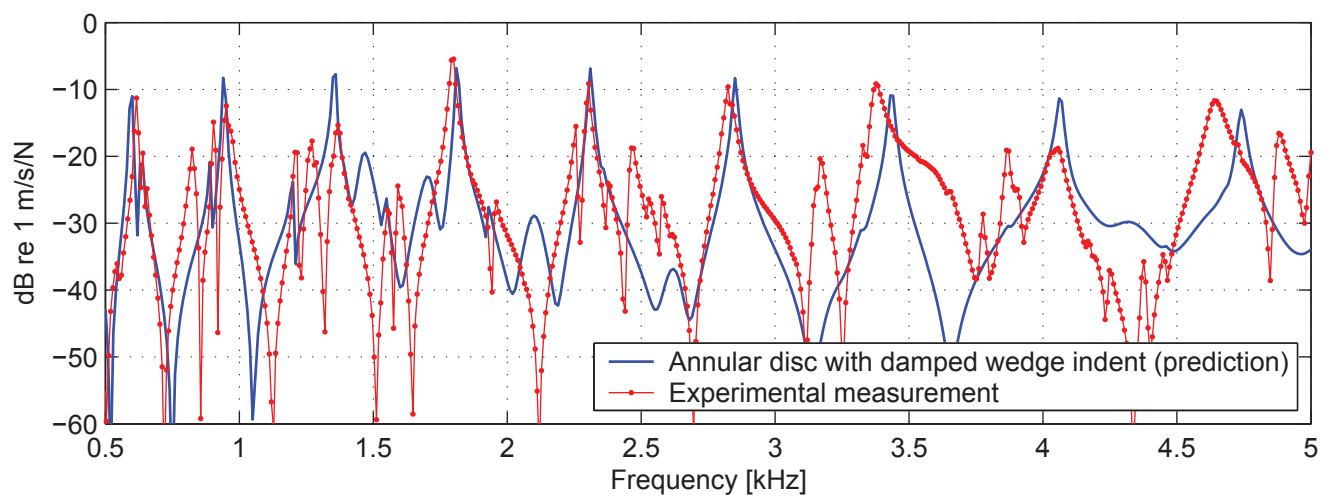

Fig. 6. Comparison of the predicted driving point mobility $\dot{w}\left(R_{m}, \theta=0, \omega\right) / p\left(R_{m}\right)$ for an annulus with a damped quadratic power-law indentation machined into it, against experimental measurements. 


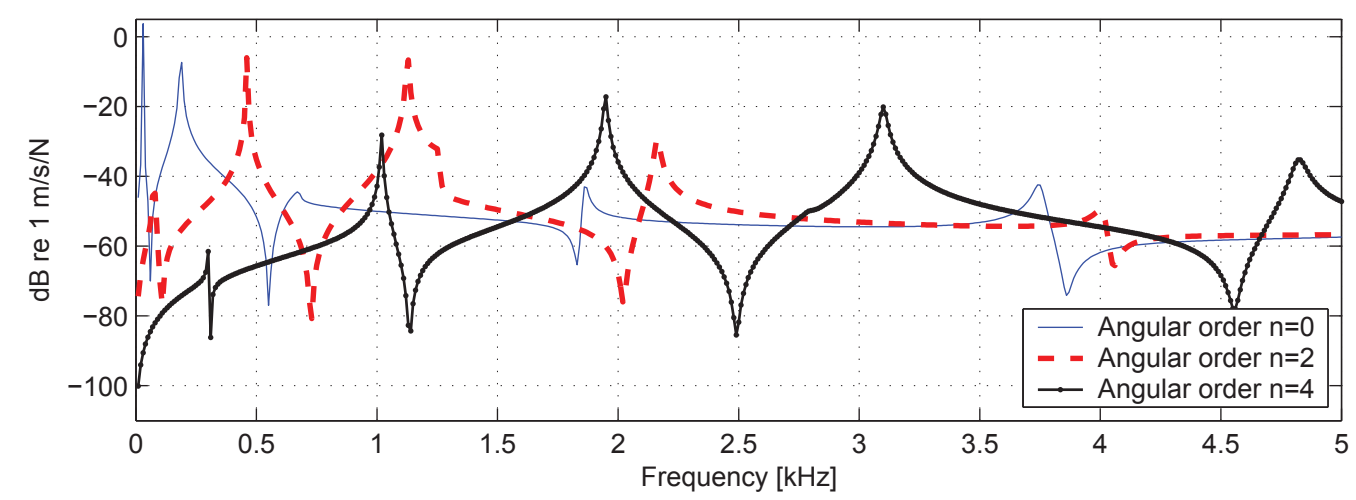

Fig. 7. Cross point mobility calculations of the composite plate, $\dot{w}\left(R_{m}, n, \omega\right) / p\left(R_{f}\right)$ for three angular orders $(n=0,2,4)$. 


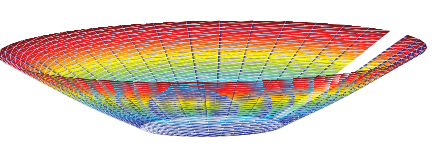

(a) $n=0$

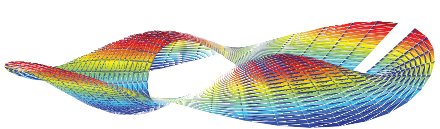

(d) $n=2$

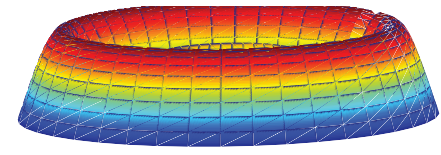

(b) $n=0$

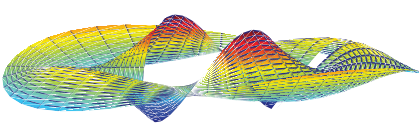

(e) $n=2$

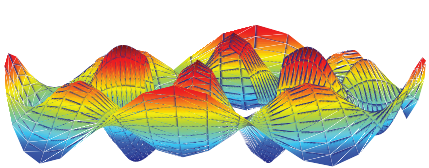

(h) $n=4$

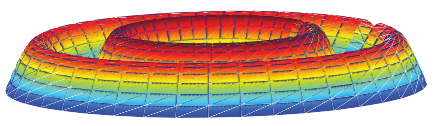

(c) $n=0$

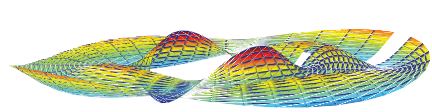

(f) $n=2$

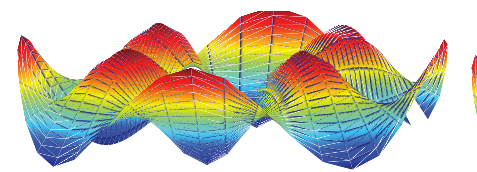

(g) $n=4$

(i) $n=4$

Fig. 8. Examples of the displacement response shapes at prescribed frequencies and angular orders; For a frequency (a) $f=0.22 \mathrm{kHz}$, (b) $f=1.85 \mathrm{kHz}$, (c) $f=3.74 \mathrm{kHz},(\mathrm{d}) f=0.49 \mathrm{kHz},(\mathrm{e}) f=1.20 \mathrm{kHz},(\mathrm{f}) f=2.20 \mathrm{kHz},(\mathrm{g})$ $f=0.98 \mathrm{kHz}$, (h) $f=1.90 \mathrm{kHz}$, (i) $f=3.10 \mathrm{kHz}$. 


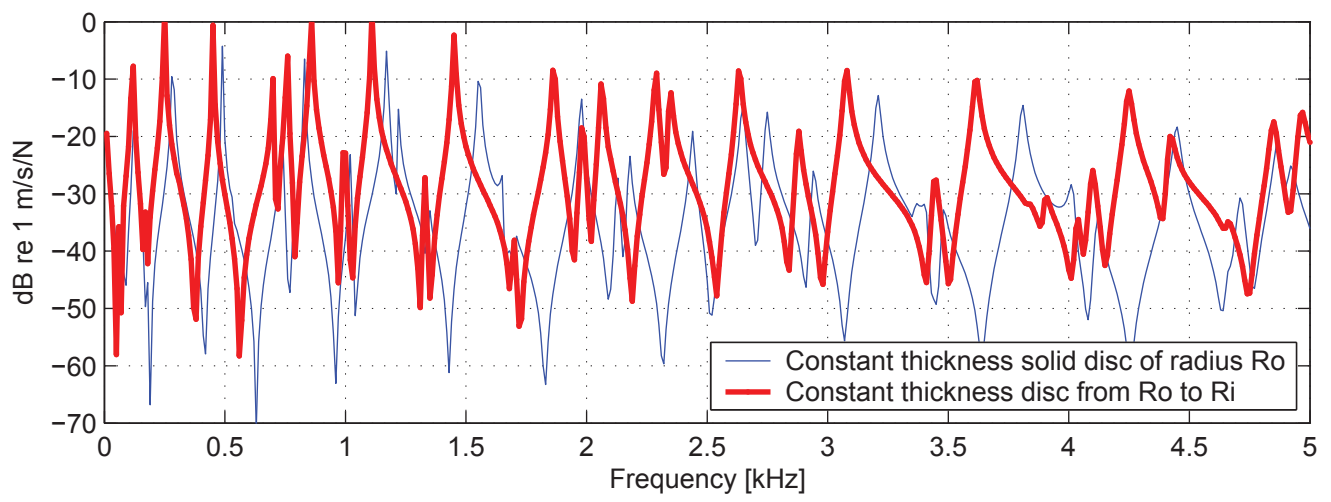

(a)

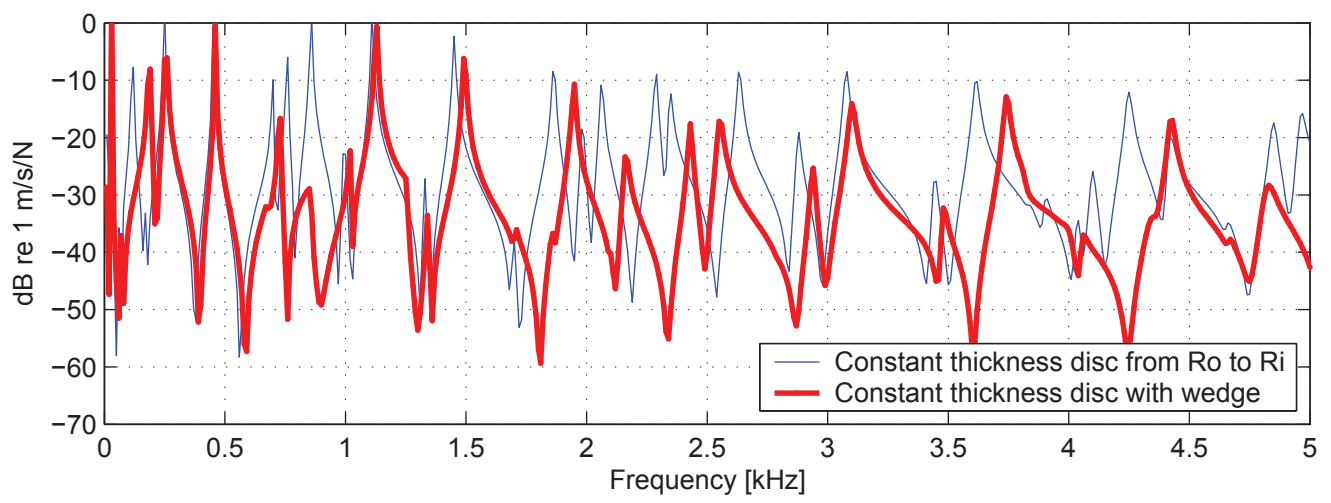

(b)

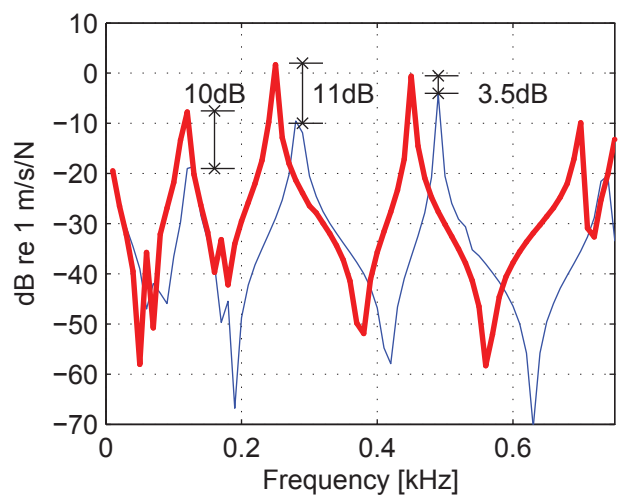

(c)

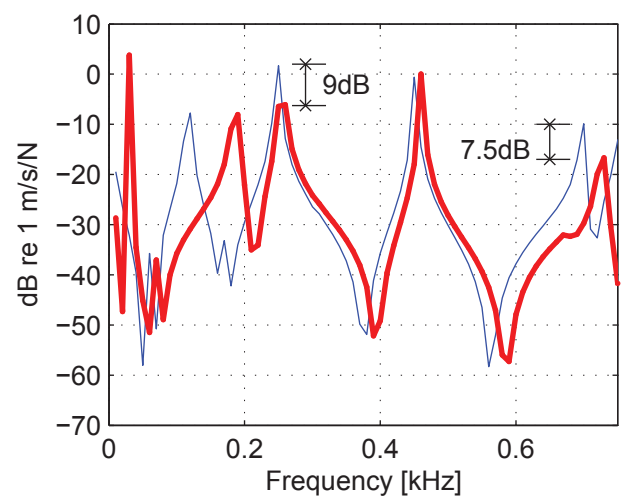

(d)

Fig. 9. Comparison of the cross point mobility calculations of three different plates after the Fourier sum over angular order has been completed, $\dot{w}\left(R_{m}, \theta=0, \omega\right) / p\left(R_{f}\right)$. Plates are a) a constant thickness plate from $r=R_{o}$ to $r=0$ against a constant thickness plate from $r=R_{o}$ to $\left.r=R_{i} ; \mathrm{b}\right)$ a constant thickness plate from $r=R_{o}$ to $r=R_{i}$ compared against a constant thickness plate from $r=R_{o}$ to $r=R_{i}$ with a profiled wedge 4 section from $R_{i}$ to $R_{t}$. The low frequency regions of these two results are shown in detail in figures c) and d) respectively 


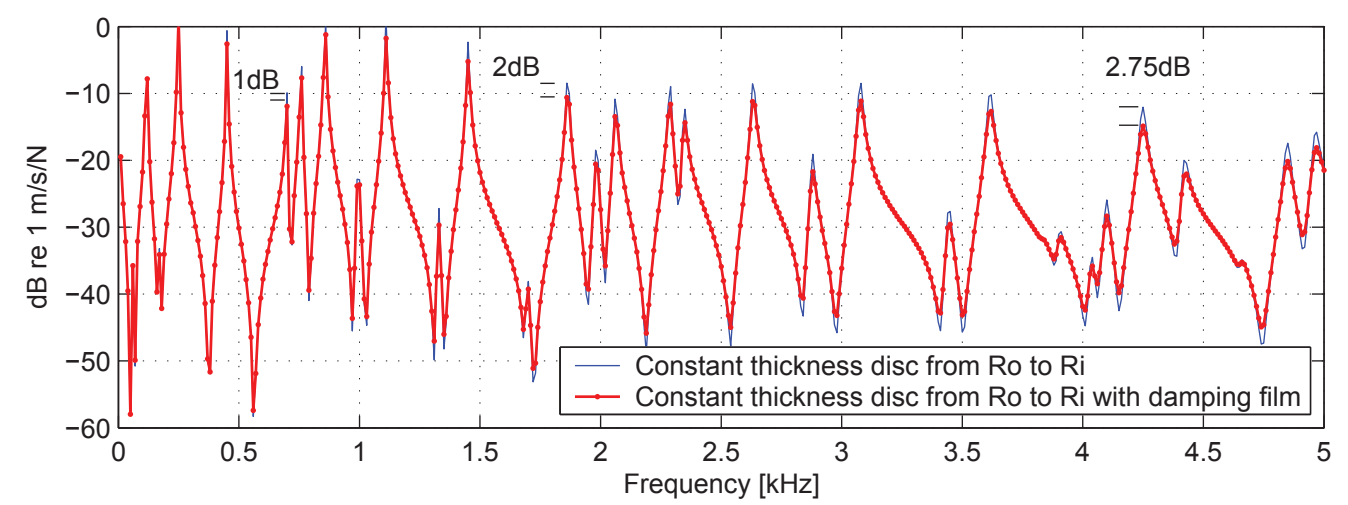

Fig. 10. Comparison of the cross point mobility calculations of two different plates, $\dot{w}\left(R_{m}, \theta=0, \omega\right) / p\left(R_{f}\right)$. Plates are a constant thickness plate from $r=R_{o}$ to $r=R_{i}$ with only inherent metal damping against the same plate covered with a layer of damping film (the composite damping coefficient is $\eta=8.45 \times 10^{-3}$ ). 


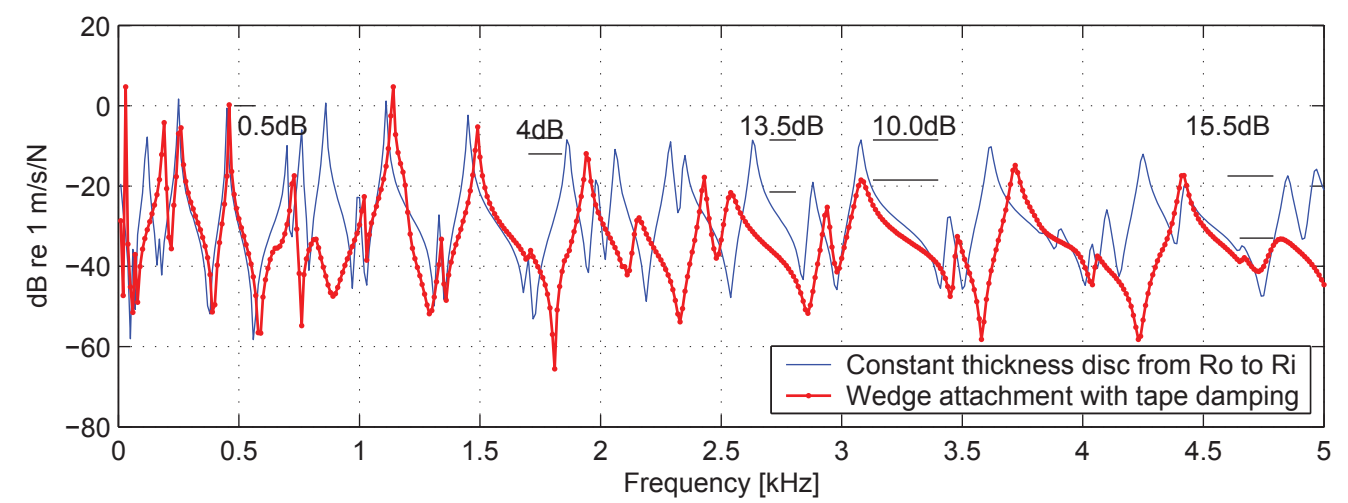

Fig. 11. Comparison of the cross point mobility calculations of two different plates, $\dot{w}\left(R_{m}, \theta=0, \omega\right) / p\left(R_{f}\right)$. Plates are a constant thickness plate from $r=R_{o}$ to $r=R_{i}$ with only inherent metal damping against the same plate with a profiled wedge section from $r=R_{i}$ to $R_{t}$ covered with a layer of damping film (the composite damping coefficient is $\eta=1.51 \times 10^{-2}$ ). 


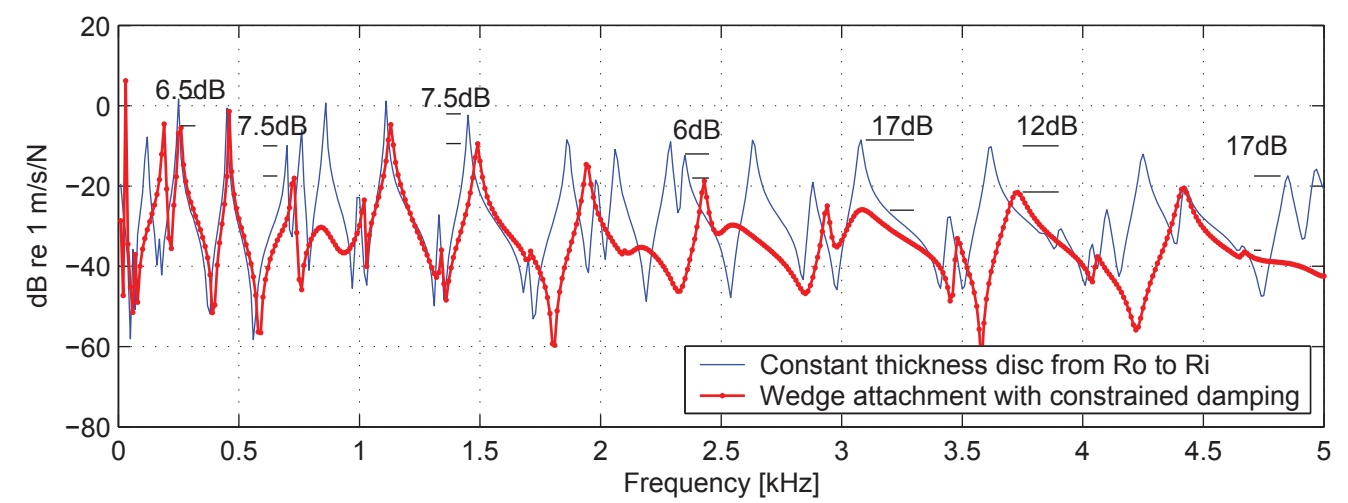

Fig. 12. Comparison of the cross point mobility calculations of two different plates, $\dot{w}\left(R_{m}, \theta=0, \omega\right) / p\left(R_{f}\right)$. Plates are a constant thickness plate from $r=R_{o}$ to $r=R_{i}$ with a profiled wedge attachment from $r=R_{i}$ to $R_{t}$ compared with the same plate when the wedge-like hole is covered with a constrained layer damping solution (the composite damping coefficient is $\eta=7.98 \times 10^{-2}$ ). 


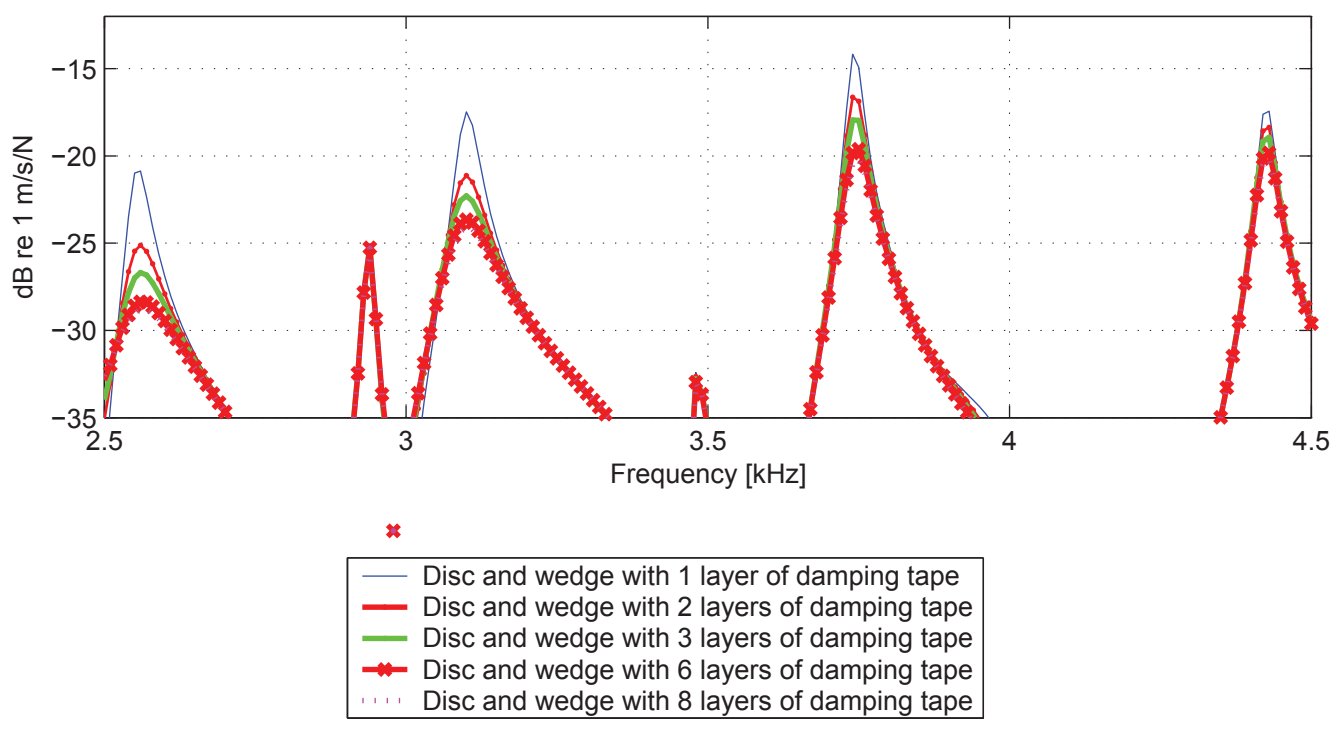

Fig. 13. Comparison of the cross point mobility calculations of the cylindrical plate with a damped wedge profile, $\dot{w}\left(R_{m}, \theta=0, \omega\right) / p\left(R_{f}\right)$. The thickness of damping layer applied to the surface is varied such that the peak amplitude response is further reduced. 


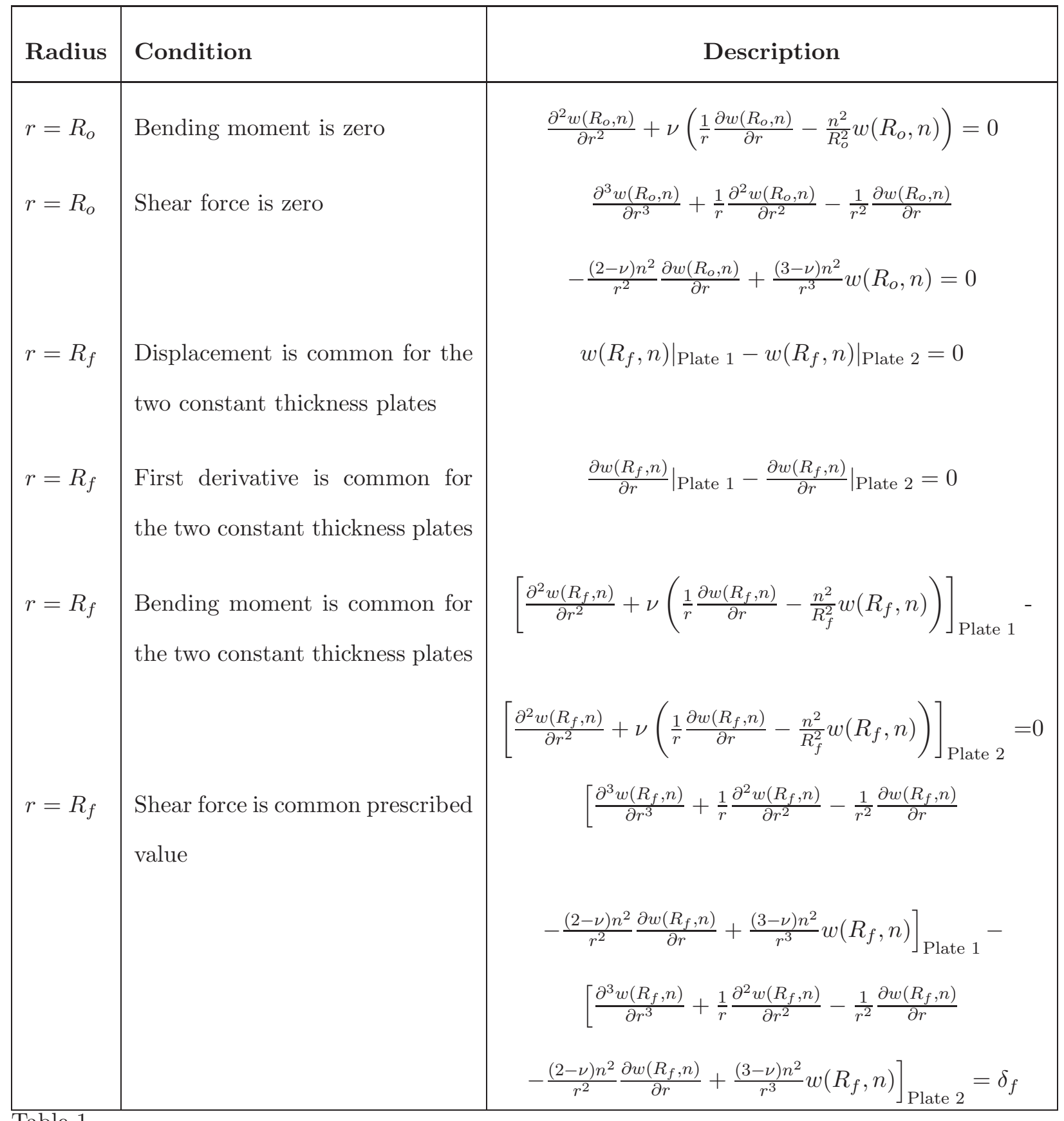

Table 1

Boundary conditions applied to the outer constant thickness plate (1) and the inner constant thickness plate (2). 


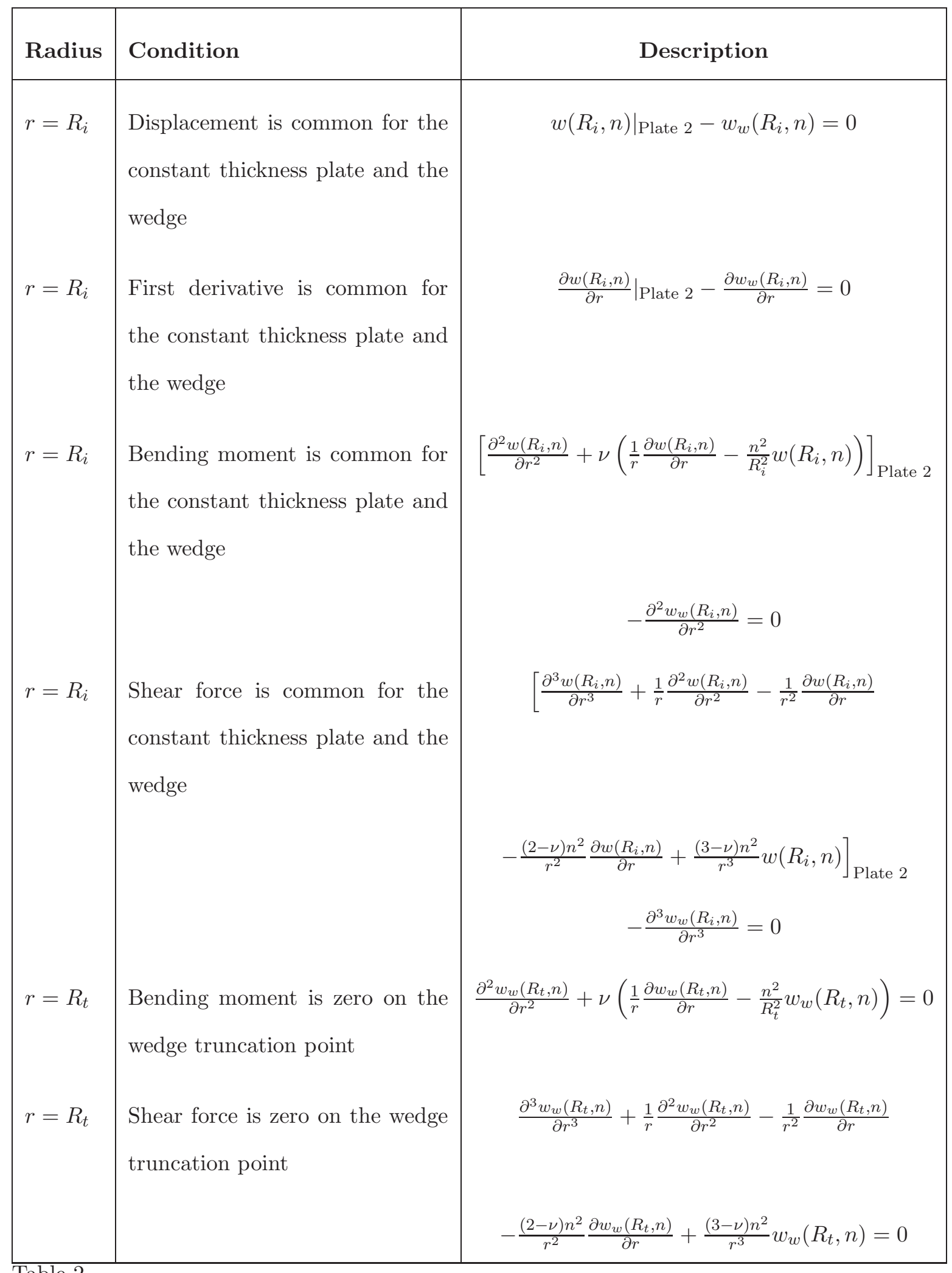

Table 2

Boundary conditions applied to the inner constant thickness plate and the wedgelike section. 


\begin{tabular}{|c|c|c|}
\hline Parameter & Notation & Value \\
\hline Young's modulus & $E$ & $190 \mathrm{GPa}$ \\
\hline Density & $\rho$ & $7800 \mathrm{~kg} / \mathrm{m}^{3}$ \\
\hline Poisson's ratio & $\nu$ & 0.30 \\
\hline Damping & $\eta$ & 0.006 \\
\hline Outer radius & $R_{o}$ & $0.30 \mathrm{~m}$ \\
\hline Forcing location & $R_{f}$ & $0.12 \mathrm{~m}$ \\
\hline Inner radius & $R_{i}$ & $0.10 \mathrm{~m}$ \\
\hline Measurement radius & $R_{m}$ & $0.10 \mathrm{~m}$ \\
\hline Truncation radius & $R_{t}$ & $0.005 \mathrm{~m}$ \\
\hline Initial thickness & $h$ & $0.005 \mathrm{~m}$ \\
\hline Damping loss factor & $\eta_{D}$ & 0.2 \\
\hline Damping layer thickness & $h_{D}$ & $0.3 \mathrm{~mm}$ \\
\hline
\end{tabular}

Table 3

Parameters of the composite plate used in the numerical predictions. 


\begin{tabular}{|l|c|}
\hline Number of layers of damping tape applied & Equivalent loss factor \\
\hline One & 0.0151 \\
Two & 0.0356 \\
Three & 0.0488 \\
Six & 0.0720 \\
Eight & 0.0814 \\
Ten & 0.0889 \\
\hline
\end{tabular}

Table 4

Equivalent loss factors for a wedge with a different number of layers of thin elastic damping tape applied to the surface. 


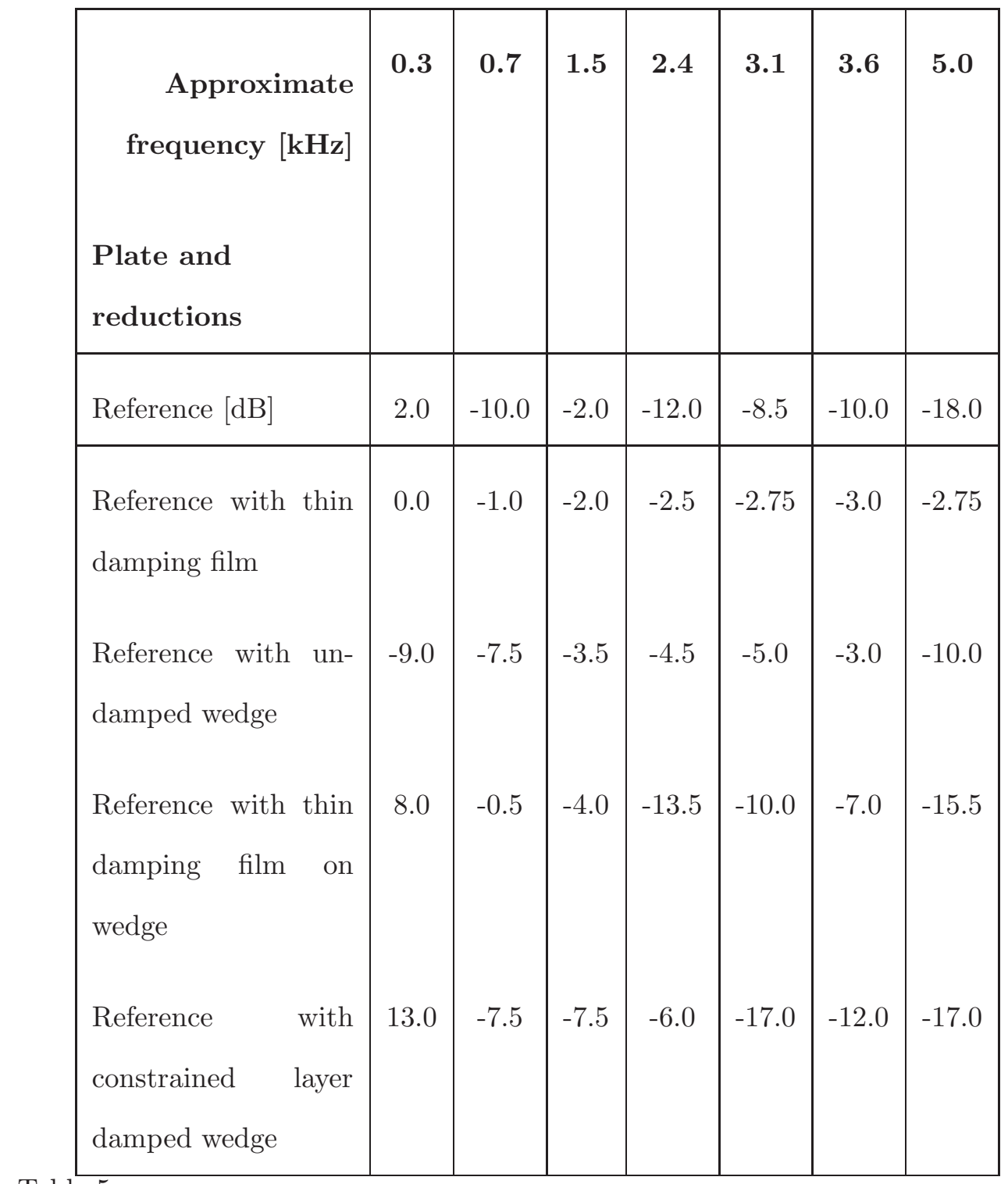

Table 5

Summary of potential reductions in plate resonance using different damping methods. The reference calculation is the cross mobility response of a constant thickness plate of outer radius $r=R_{o}$ and inner radius $R_{i}$. The subsequent approximate reductions in $\mathrm{dB}$ re $1 \mathrm{~m} / \mathrm{s} / \mathrm{N}$ are given for the reference plate covered with damping film, the reference plate with an undamped and damped wedge section and finally with a constrained layer damping applied to the wedge section. 